\title{
Copper-Catalyzed Aerobic Cascade Oxidative Coupling/Cyclization for the Construction of 1,4-Dihydropyridine Derivatives
}

Zhi-Qiang Zhu, ${ }^{* \dagger,+}$ Zong-Bo Xie, ${ }^{\dagger, t}$ Zhang-Gao Le*, ${ }^{*,+}$

†Jiangxi 2011 Joint Center for the Innovative Mass Spectrometry and Instrumentation and School of Chemistry, Biology and Material Science, East China University of Technology, Nanchang 330013, P. R. China

*E-mail: zhuzq@ecit.cn; *E-mail: zhgle@ecit.edu.cn

\section{Supporting Information}

Contents:

1. Optimization of the Reaction Conditions.....................................S2

2. ${ }^{1} \mathrm{H}$ NMR, ${ }^{13} \mathrm{C}$ NMR and HR-MS Spectra of Products........................S4 


\section{Optimization of the Reaction Conditions}

Table S1. Screening of transition metal catalysts ${ }^{\mathrm{a}}$

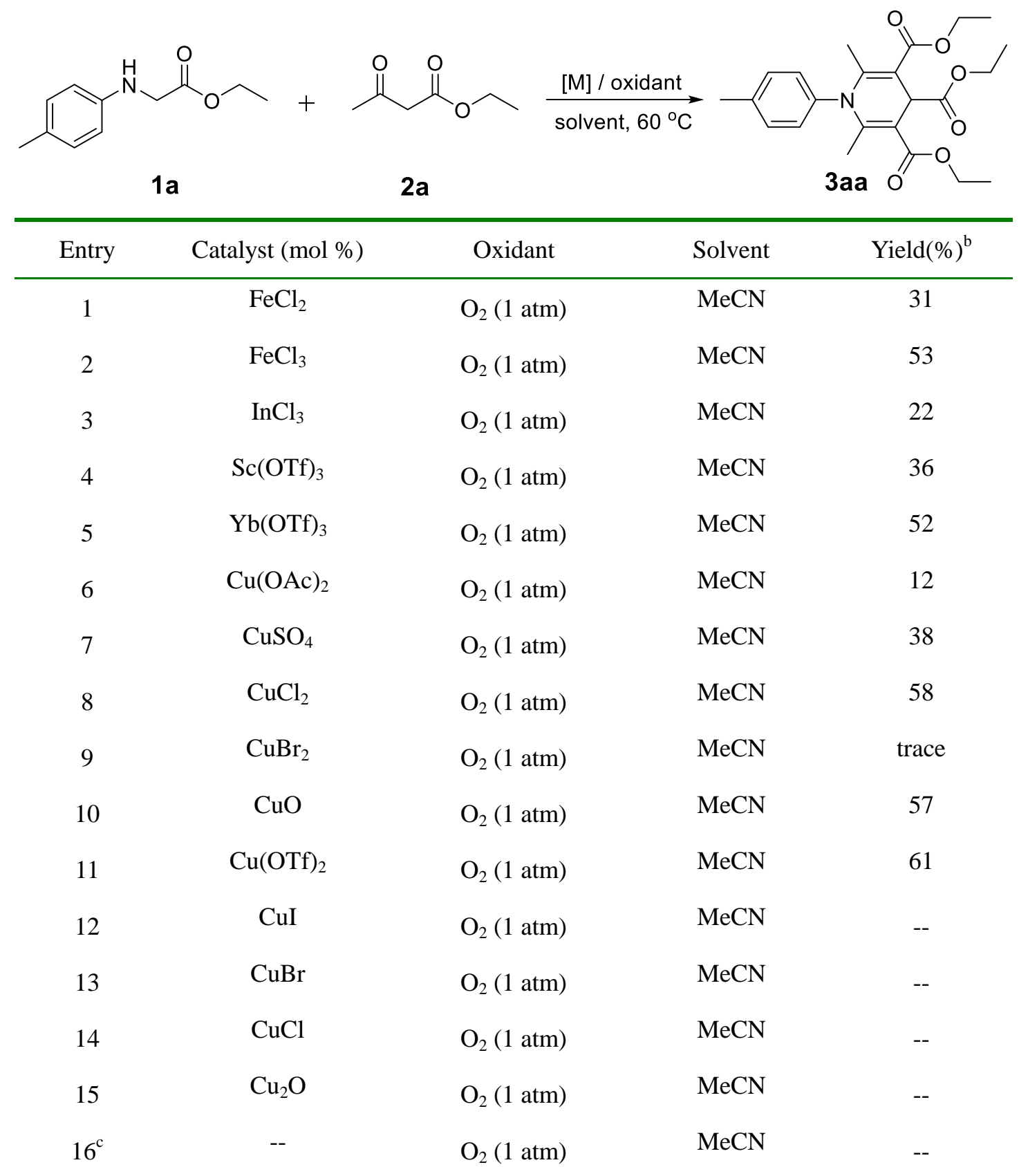

(a) Reaction conditions: 1a $(0.2 \mathrm{mmol}), \mathbf{2 a}(0.44 \mathrm{mmol})$, catalyst $(10 \mathrm{~mol} \%)$, solvent $(1 \mathrm{~mL})$ at 60 ${ }^{\circ} \mathrm{C}$ under $\mathrm{O}_{2}(1 \mathrm{~atm})$ or oxidant (2 equiv) for $12 \mathrm{~h}$. (b) Isolated yields based on 1a. (c) In the absence of a catalyst. 
Table S2. Screening of oxidants, solvents, temperatures, etc. $^{\text {a }}$

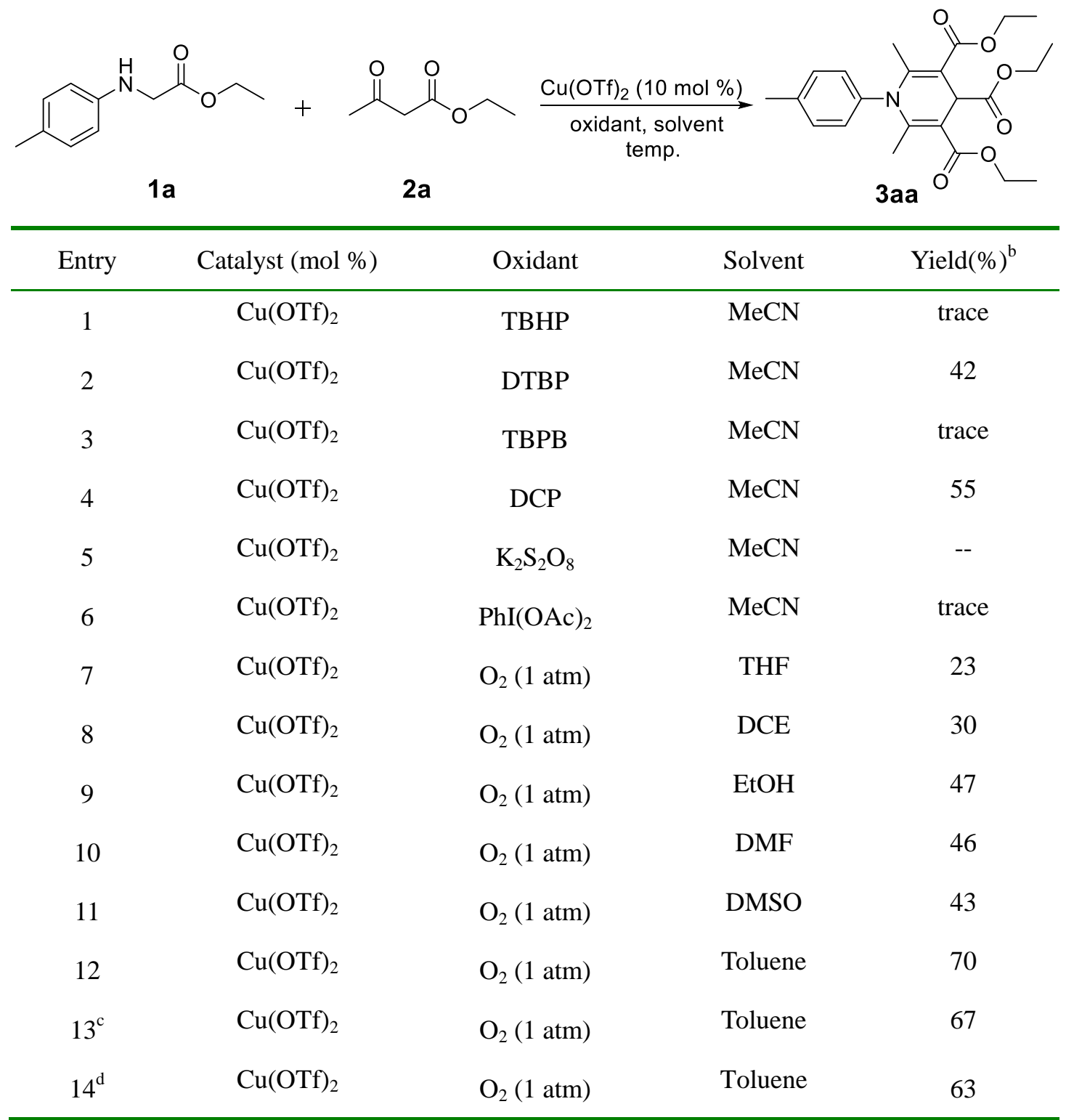

(a) Reaction conditions: 1a $(0.2 \mathrm{mmol}), \mathbf{2 a}(0.44 \mathrm{mmol}), \mathrm{Cu}(\mathrm{OTf})_{2}(10 \mathrm{~mol} \%)$, solvent $(1 \mathrm{~mL})$ at $60{ }^{\circ} \mathrm{C}$ under $\mathrm{O}_{2}(1 \mathrm{~atm})$ or oxidant (2 equiv) for $12 \mathrm{~h}$. (b) Isolated yields based on 1a. (c) At $40{ }^{\circ} \mathrm{C}$ for $24 \mathrm{~h}$. (d) At $80{ }^{\circ} \mathrm{C}$. 


\section{2. ${ }^{1} \mathrm{H}$ NMR, ${ }^{13} \mathrm{C}$ NMR and HR-MS Spectra of Products}

${ }^{1}$ H NMR Spectra of triethyl 1,4-dihydro-2,6-dimethyl-1- $p$-tolylpyridine-3,4,5-tricarboxylate 3aa

$000^{\circ} 0^{-}$

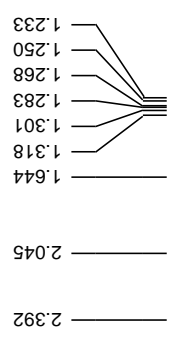

\&Lเ॰

$6+1 . t 7$

191.7

$\angle 91 \cdot 7 \rightarrow$

$1 \angle L^{\circ} \circ$
$6 \angle L^{\circ} \circ$

$681^{\circ} \mathrm{C}$

$902 ๋$

○๖ट゙॰ -

6๖で —

8งट゙॰ -

$\angle 9)^{\circ} \circ$
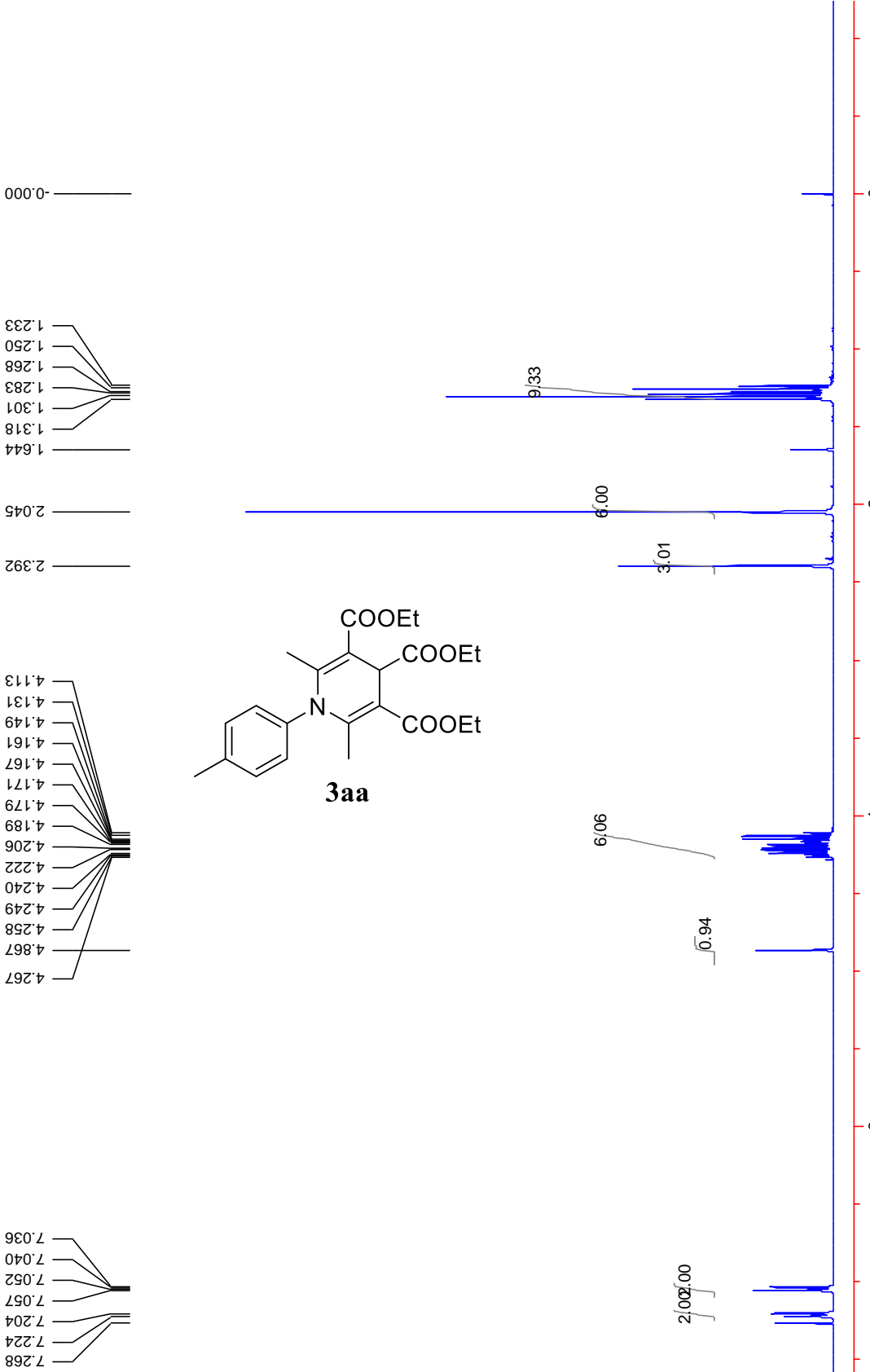

б.

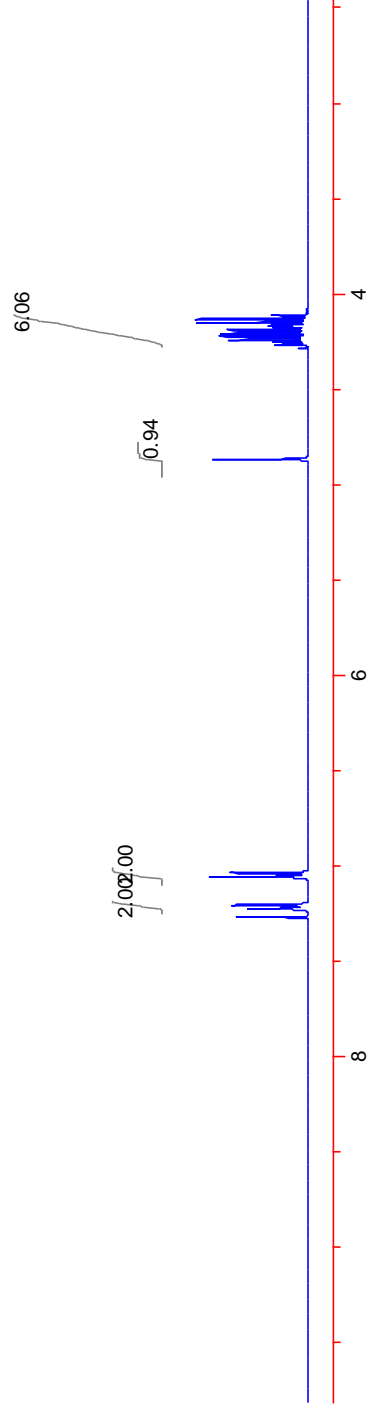


${ }^{1} \mathrm{H}$ NMR Spectra of triethyl 1,4-dihydro-1-(4-methoxyphenyl)-2,6-dimethylpyridine3,4,5-tricarboxylate $\mathbf{3 b a}$

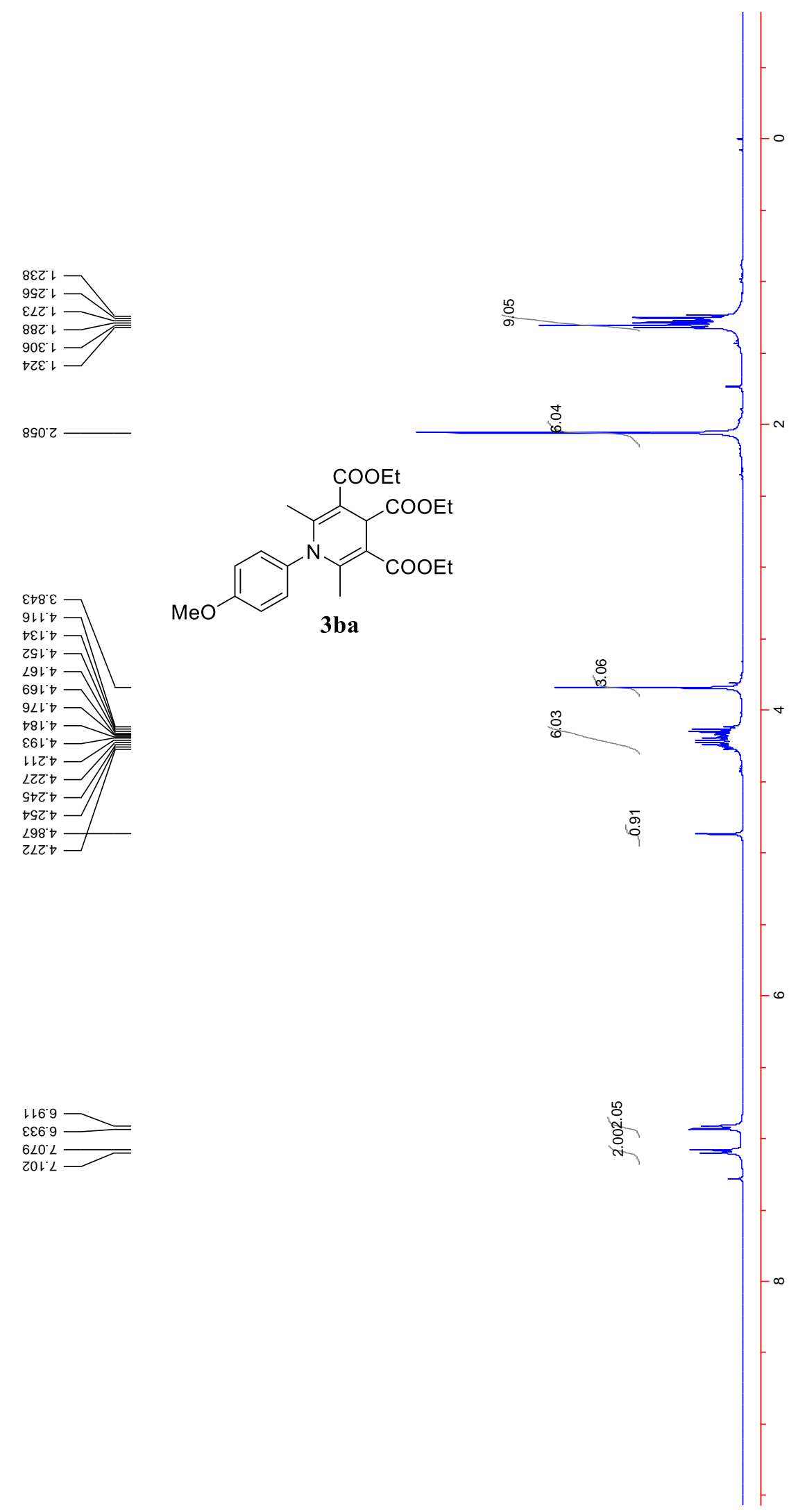


${ }^{1}$ H NMR Spectra of triethyl 1,4-dihydro-2,6-dimethyl-1- $m$-tolylpyridine-3,4,5-tricarboxylate 3ca

$000^{\circ} 0^{-}$
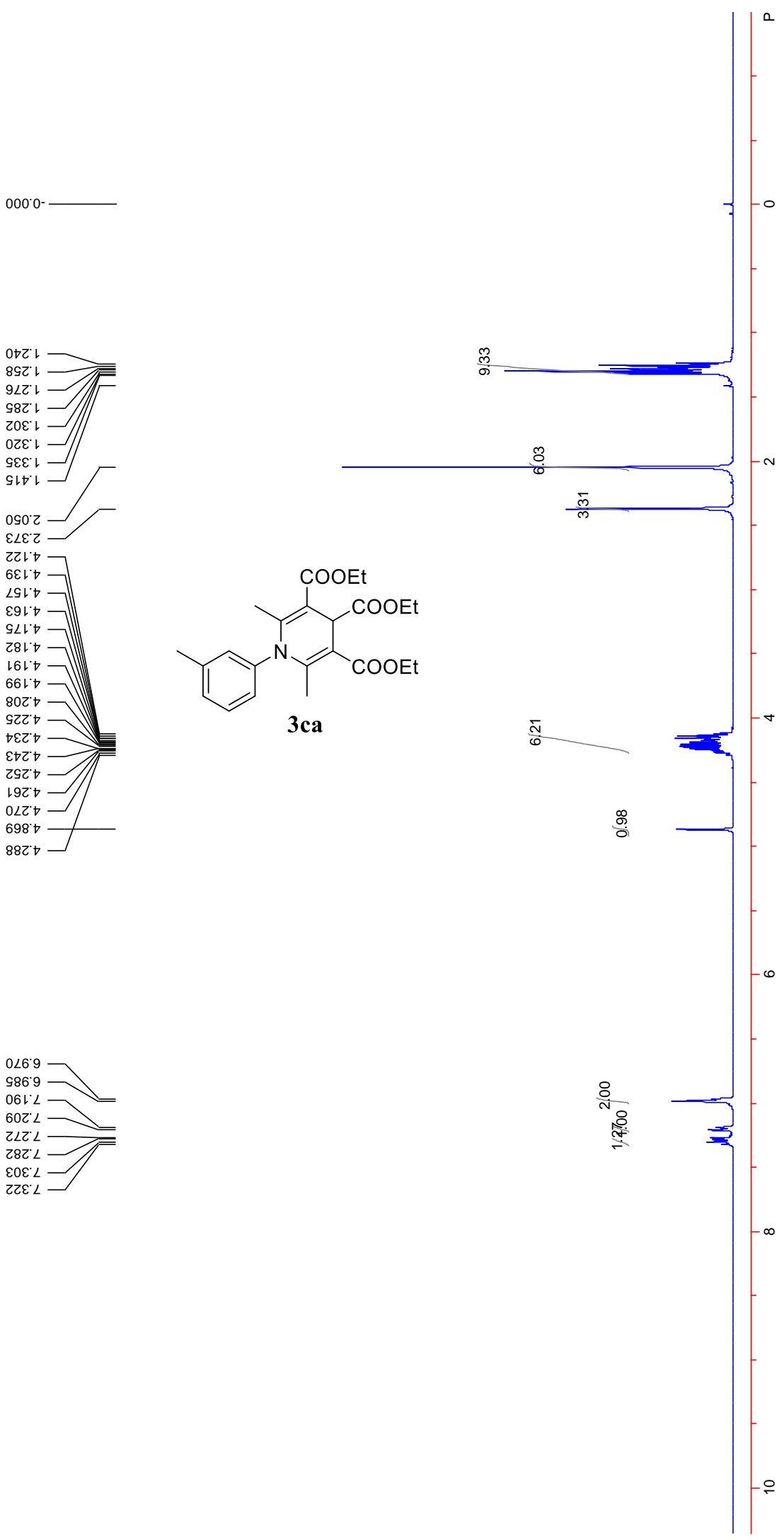
${ }^{13}$ C NMR Spectra of triethyl 1,4-dihydro-2,6-dimethyl-1-m-tolylpyridine-3,4,5-tricarboxylate 3 ca

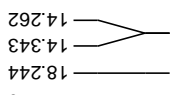

$0+乙 เ 2$

$62+0 t$

$90+09 \longrightarrow$

$0 \varepsilon \angle \cdot 9 L \longrightarrow$

$\angle 9 \varepsilon^{\circ} \angle L$

$710.01-$

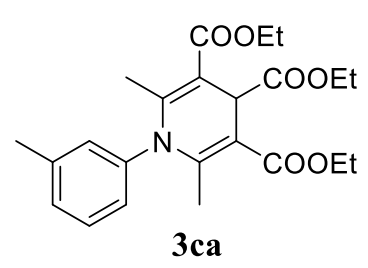

t9t: LZL 3ca

St+62!

St+62!

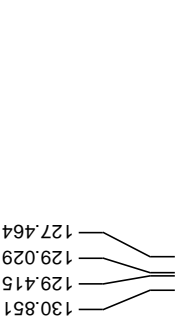

2SG. $6 \varepsilon+\square$

$888^{\circ} 8 \mathrm{t1}$

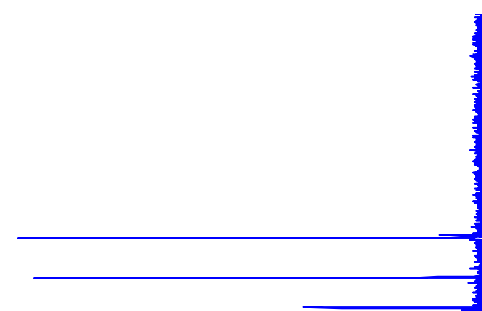

$9<9^{\circ}<91$

५68 $\varepsilon \angle\llcorner$ 
HR-MS Spectra of triethyl 1,4-dihydro-2,6-dimethyl-1-m-tolylpyridine-3,4,5-tricarboxylate 3ca

ฐ্ণ
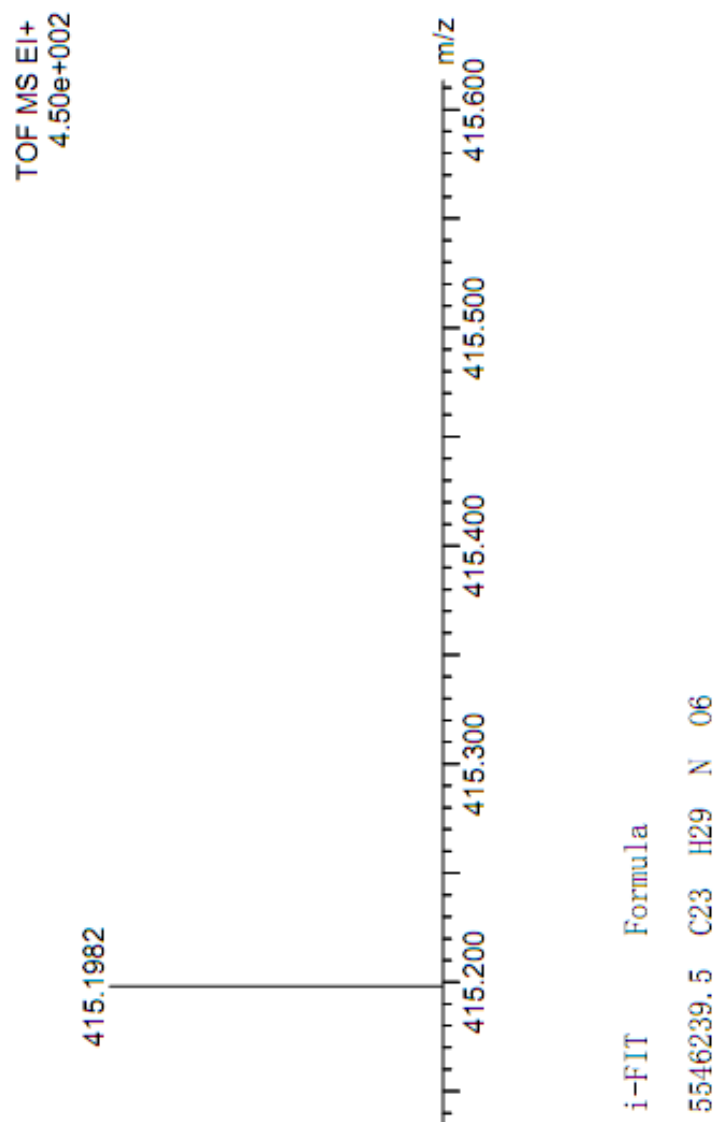

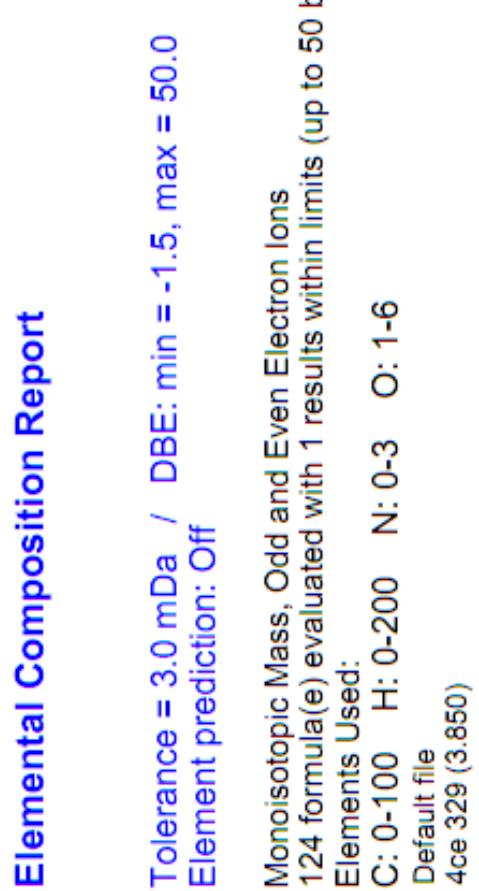

ம0 빔용

$\stackrel{\circ}{\circ}$ 종

ำ

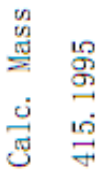

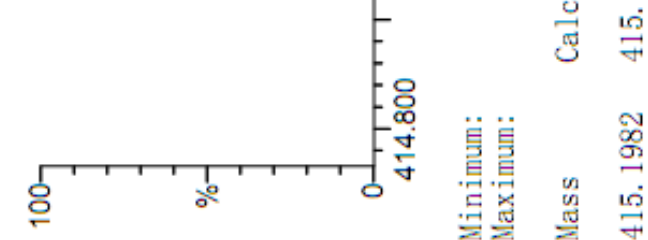


${ }^{1}$ H NMR Spectra of triethyl 1,4-dihydro-2,6-dimethyl-1-phenylpyridine-3,4,5-tricarboxylate 3da

$000^{\circ} 0^{-}$

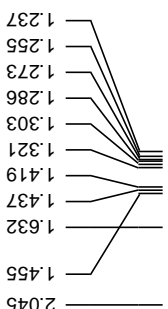

6เト'

sqL't

991.7

SLI'

$\neg 81^{\circ} t$

$\varepsilon 6 r^{\circ} \downarrow$

こOZ'

เレで

๑Ъでち

घ९ट๐ -

เ9ट十

G $\angle 8^{\circ} \triangleright$

เUZ๐<smiles>CCOC(=O)C1=C(C)N(c2ccccc2)C(C(=O)OCC)=C(C(=O)OCC)C1C(=O)OCC</smiles>
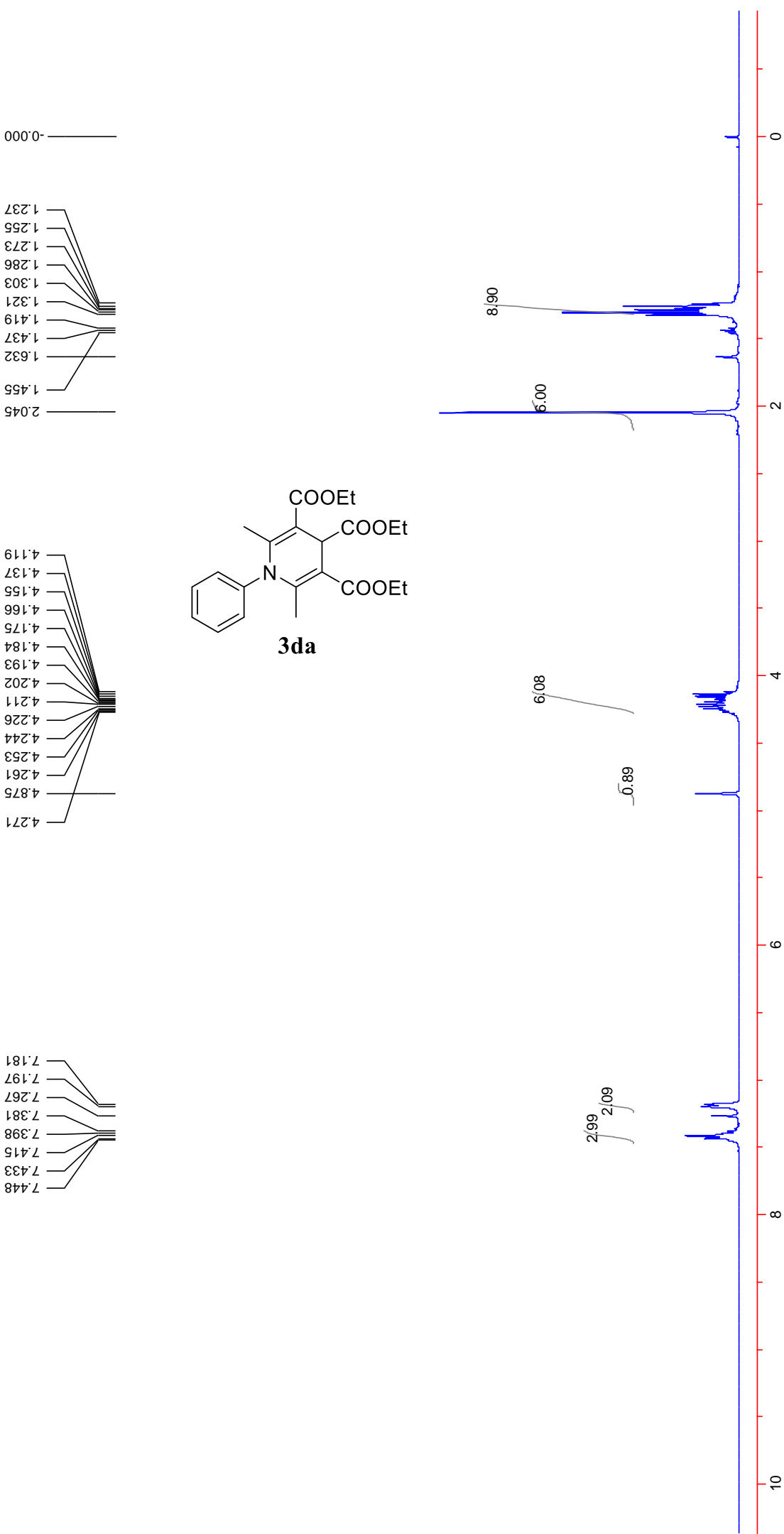
${ }^{1}$ H NMR Spectra of triethyl 1-([1,1'-biphenyl]-4-yl)-2,6-dimethyl-1,4-dihydropyridine-3,4,5-tricarboxylate 3ea<smiles>CCOC(=O)C1=C(C)N(c2ccc(-c3ccccc3)cc2)C(C)=C(C(=O)OCC)C1C(=O)OCC</smiles>
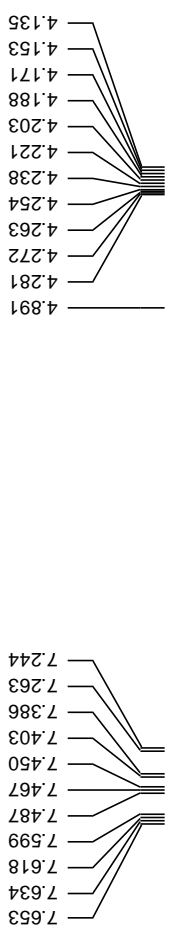
$\infty$

$\angle 8 Z+$

$96 Z^{\prime}+$

$\varepsilon\left\llcorner\varepsilon^{\circ}+\right.$

$\angle O L^{2} \mathrm{C}$

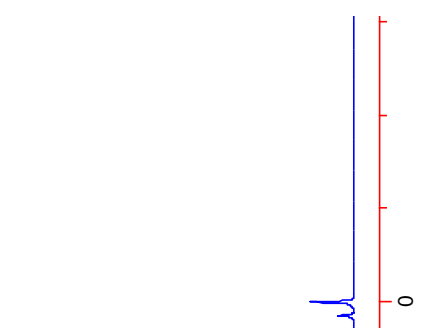

$168^{\circ} \mathrm{t}$

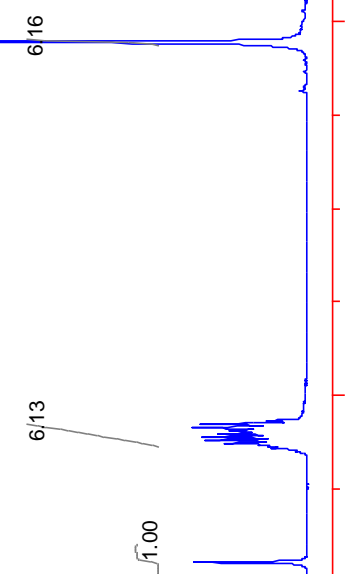

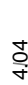

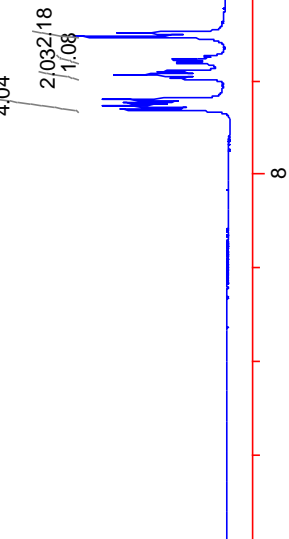


${ }^{13}$ C NMR Spectra of triethyl 1-([1,1'-biphenyl]-4-yl)-2,6-dimethyl-1,4-dihydropyridine-3,4,5-tricarboxylate 3ea

$6 S L \cdot \varepsilon t-$
$928 \cdot \varepsilon+-$
SLL'Lt

$\varepsilon \angle 96 \varepsilon$<smiles>CCOC(=O)C1=C(C)N(c2ccc(-c3ccccc3)cc2)C(C(=O)OCC)=C([Al])C1C(=O)OCC</smiles>

$\nabla t 969$

$0+1.09 \longrightarrow$

$202 \cdot 9 L \longrightarrow$

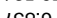

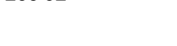

† 6001

$8 \varepsilon 9 \cdot 92$ เ

$16 \varepsilon \angle Z \vdash$

9Gt LZL

0हt. $8 \mathrm{LL}$

ง9z $0 \varepsilon$ -

0५8 $8 \varepsilon$ เ

เGZ $6 \varepsilon 1$

0งเเทト

$\angle 8 \mathrm{C}^{\circ} \mathrm{tr}$
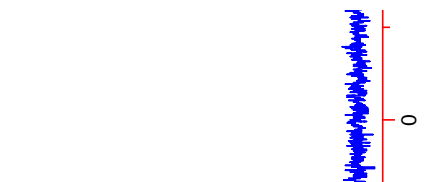

$700<91$

$9 \varepsilon \varepsilon \varepsilon\llcorner$

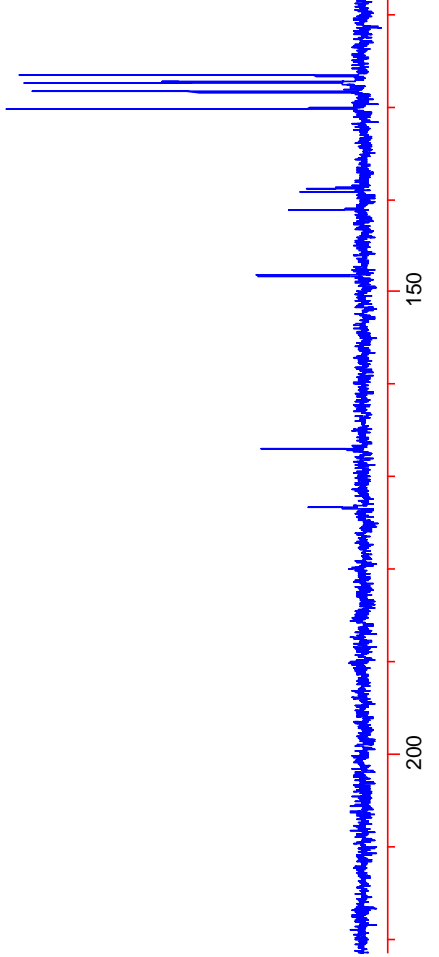


HR-MS Spectra of triethyl 1-([1,1'-biphenyl]-4-yl)-2,6-dimethyl-1,4-dihydropyridine3,4,5-tricarboxylate 3ea

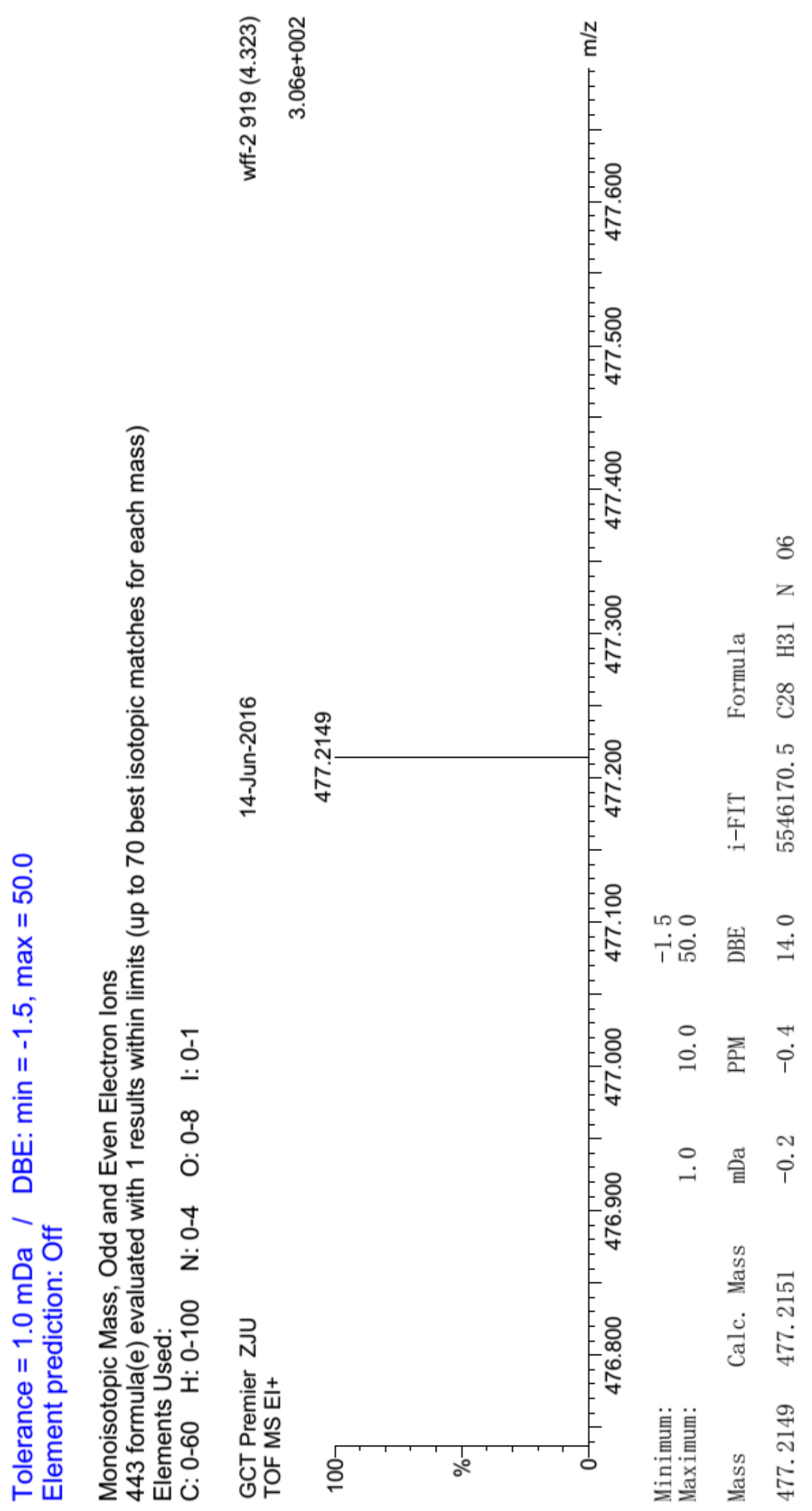


${ }^{1} \mathrm{H}$ NMR Spectra of triethyl 1-(4-chlorophenyl)-1,4-dihydro-2,6-dimethylpyridine3,4,5-tricarboxylate 3fa

$0000^{\circ-}$

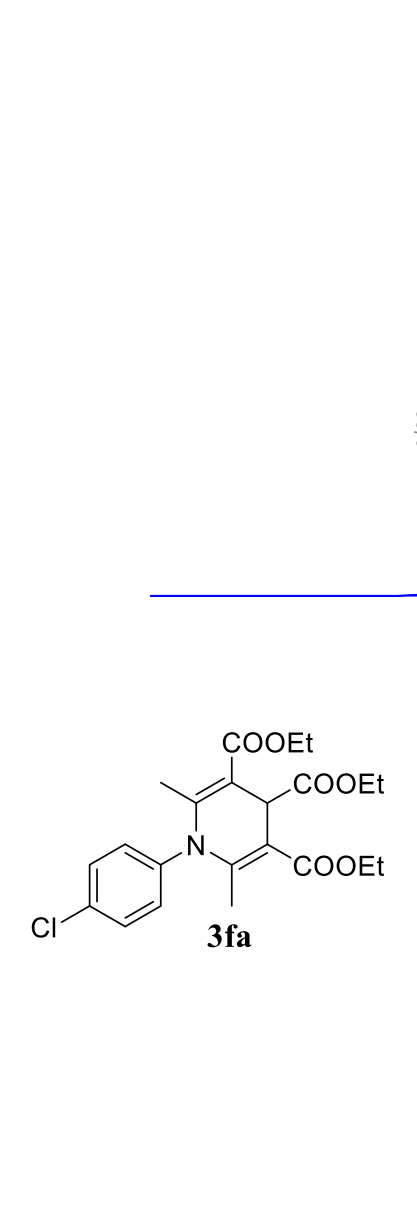

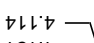

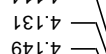

991.7

$\checkmark \angle L^{\circ} \mathrm{C}$

$\varepsilon$
$26+5$

$0 เ \check{t} \ni$

$\angle Z Z^{\prime} \downarrow$

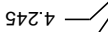

七Sट $\downarrow-$

घ9ट゙॰ -

0९8 $\bullet$

¿८टं॰

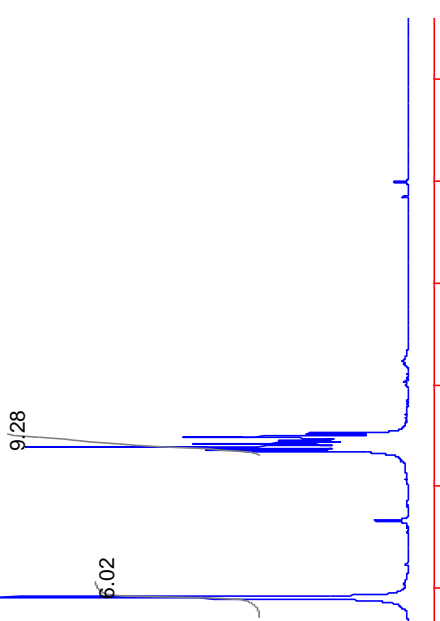

StILL
$991 \cdot L$
$Z L Z L-$
LOTL
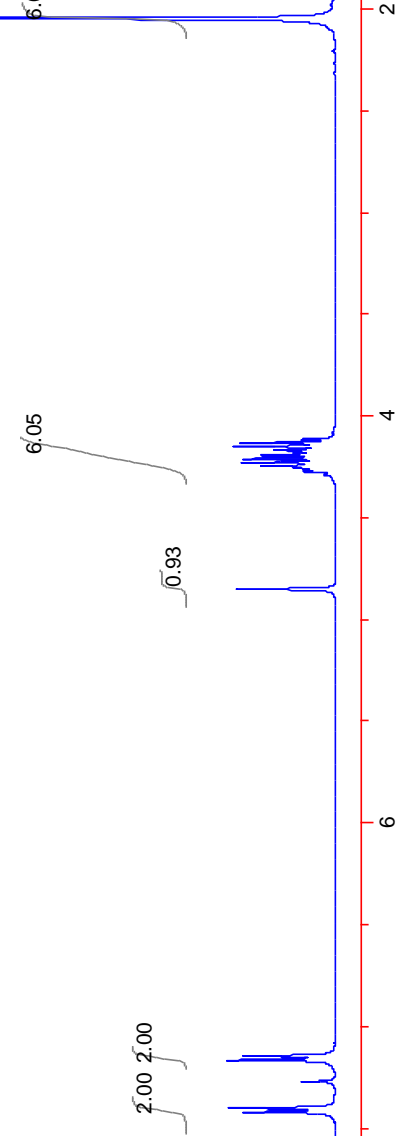

ट己t॰

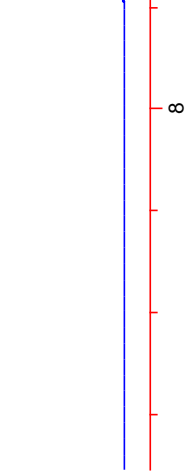


${ }^{1}$ H NMR Spectra of 3,5-diethyl 4-methyl 1,4-dihydro-2,6-dimethyl-1-p-tolylpyridine-

\section{3,4,5-tricarboxylate 3ga}

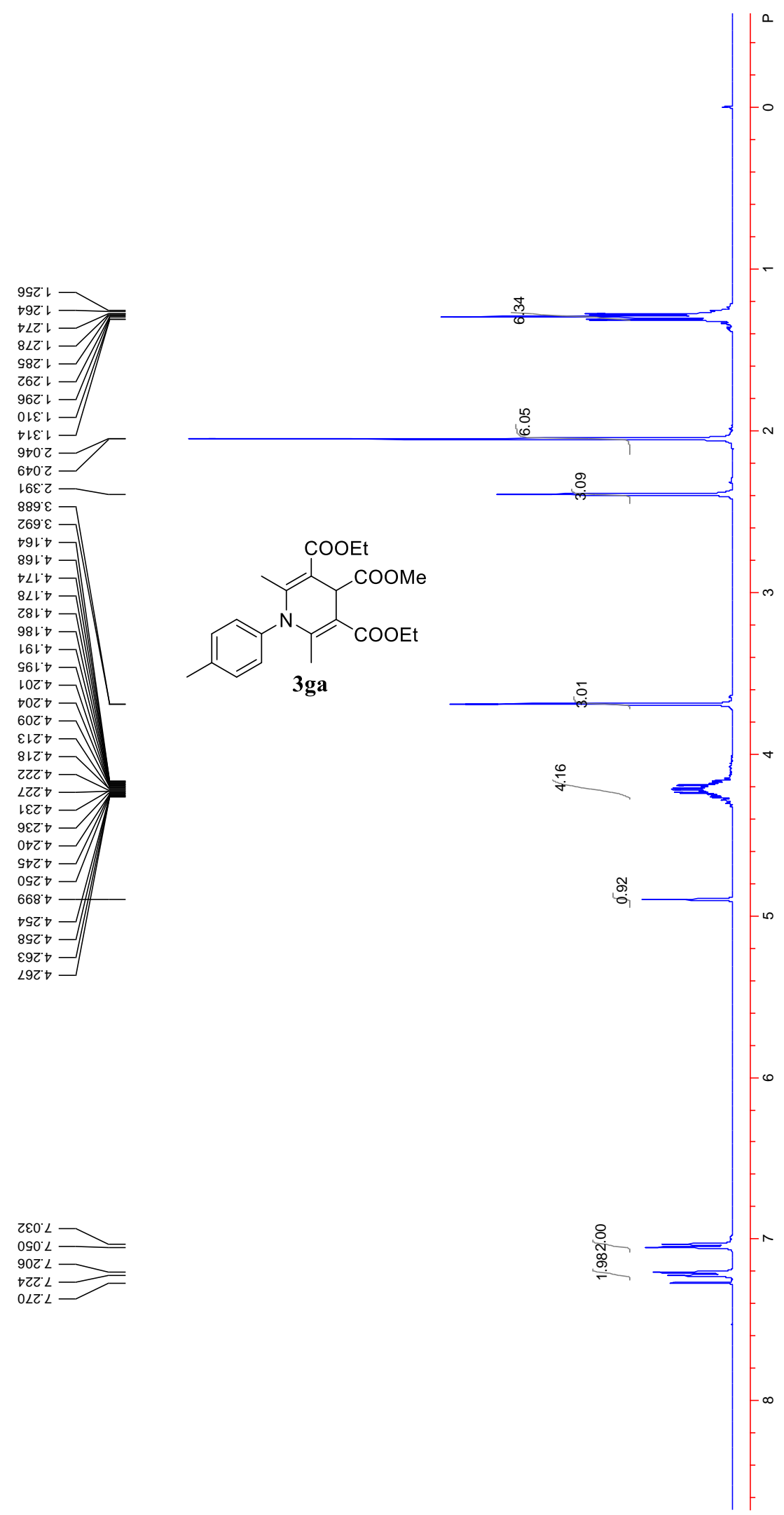


${ }^{13} \mathrm{C}$ NMR Spectra of 3,5-diethyl 4-methyl 1,4-dihydro-2,6-dimethyl-1- $p$-tolylpyridine3,4,5-tricarboxylate 3ga

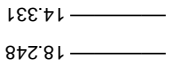

$191 \cdot 12$

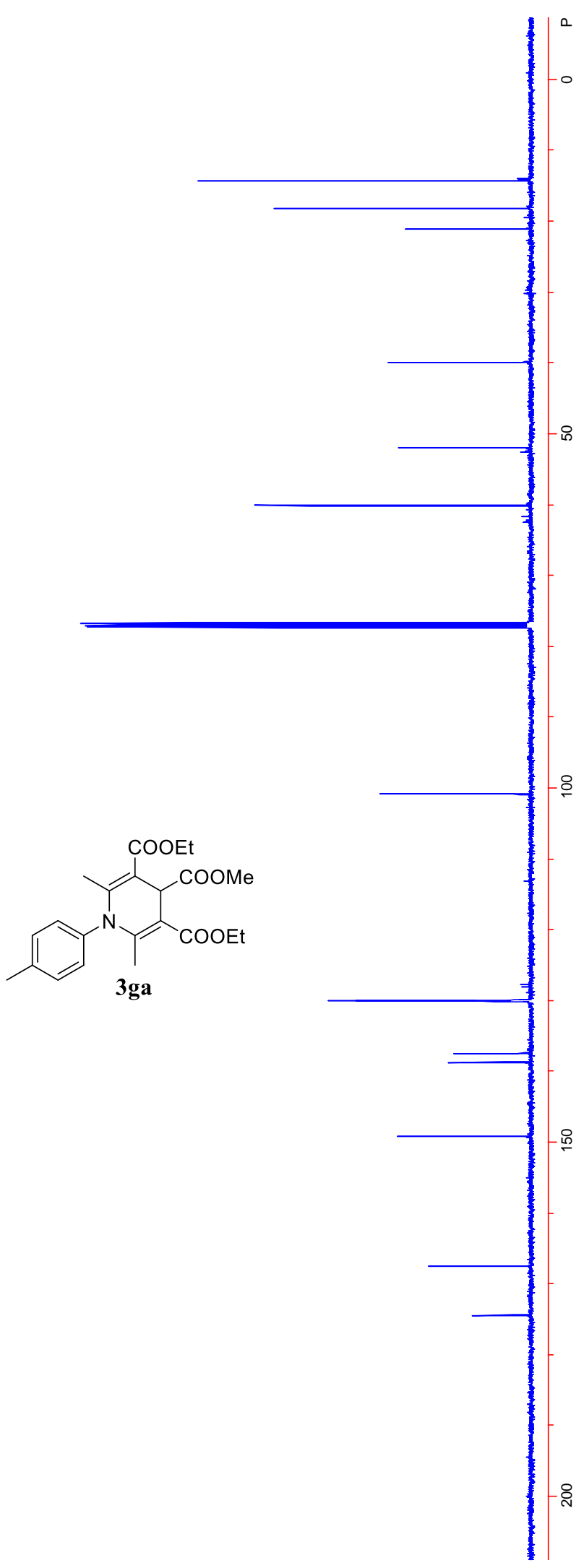

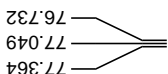

$\angle 88^{\circ} 00 \mathrm{~L}$

986.19

s9เ:09

8866 С

$060.0 \varepsilon+\longrightarrow$

こカt LEL -

$\pitchfork ट L \cdot 8 \varepsilon$

$69 ! 6$ เ

$\rightarrow 0 S^{\circ} \angle 91$

6ยヤ゙ヤடト 
HR-MS Spectra of 3,5-diethyl 4-methyl 1,4-dihydro-2,6-dimethyl-1-p-tolylpyridine3,4,5-tricarboxylate 3ga

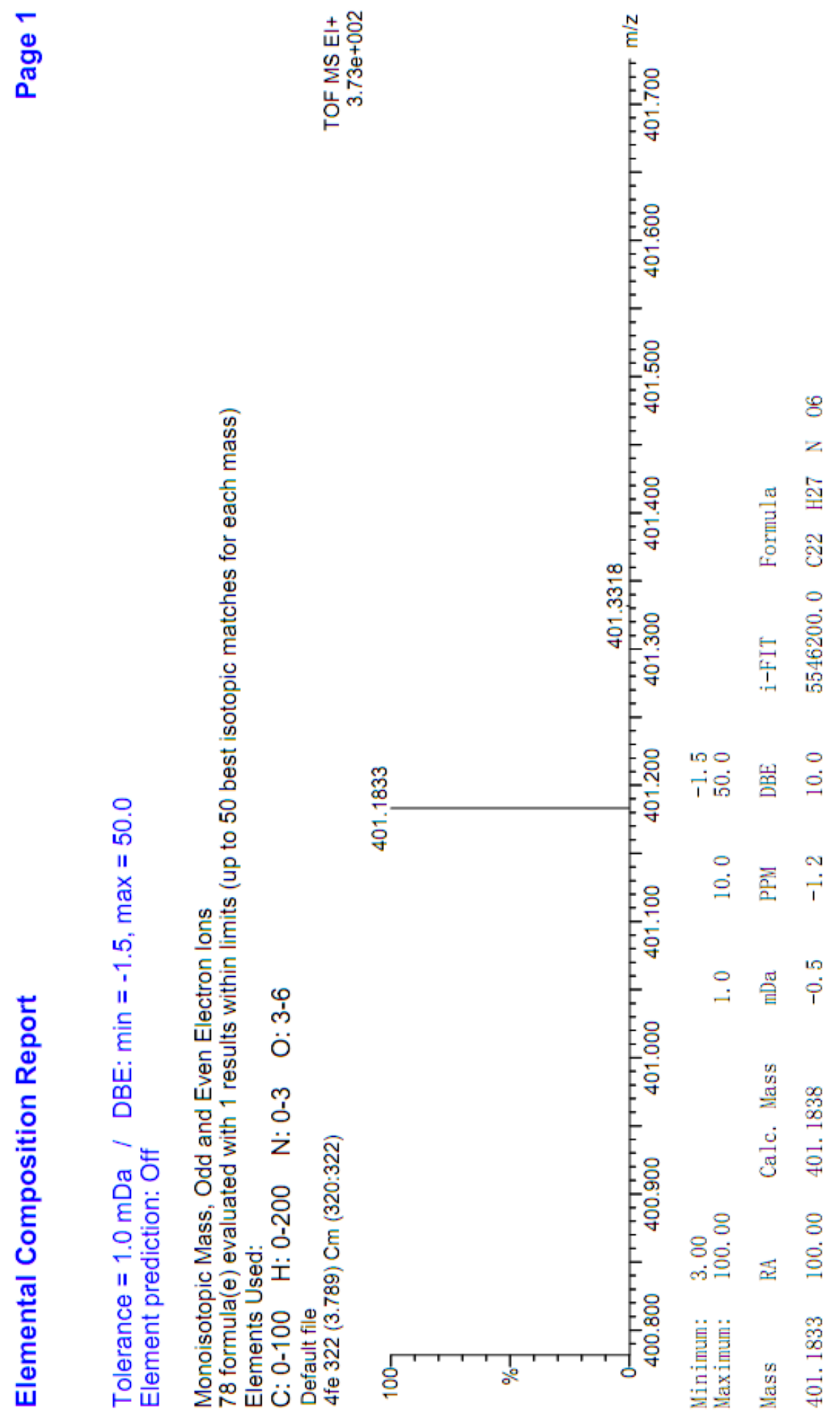


${ }^{1}$ H NMR Spectra of 3,5-diethyl 4-isopropyl 1,4-dihydro-2,6-dimethyl-1-p-tolylpyridine-3,4,5-tricarboxylate 3 ha

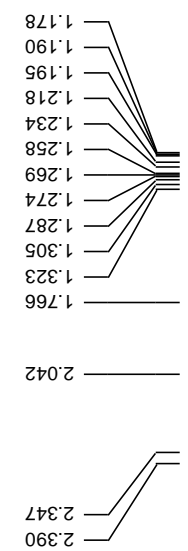

$99+\circ$

s91.t 7

$\forall \angle L \circ$

$\varepsilon 8+7 \rightarrow$

$16 L^{\circ}+1$

เОZ゙

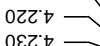

$0 \varepsilon Z^{\circ} \downarrow$

$8 \triangleright z+\downarrow$

99ट๋

g9z"

$618 t$

(

6960

$\$ 86 \mathrm{t}$

000.9

910.9
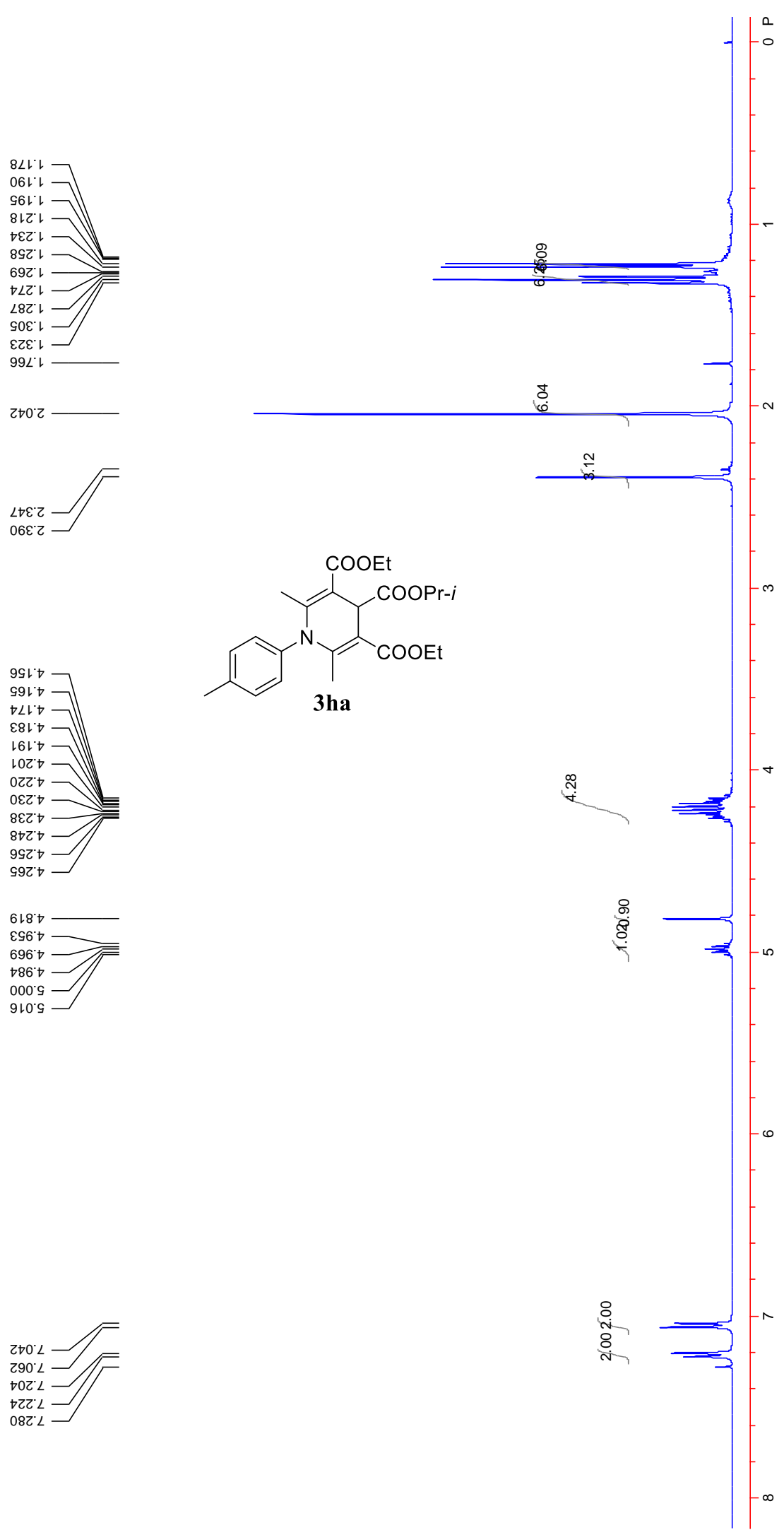
${ }^{13} \mathrm{C}$ NMR Spectra of 3,5-diethyl 4-isopropyl 1,4-dihydro-2,6-dimethyl-1-p-tolylpyridine-3,4,5-tricarboxylate $\mathbf{3 h a}$

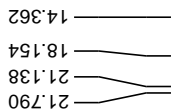

s8z 0 t

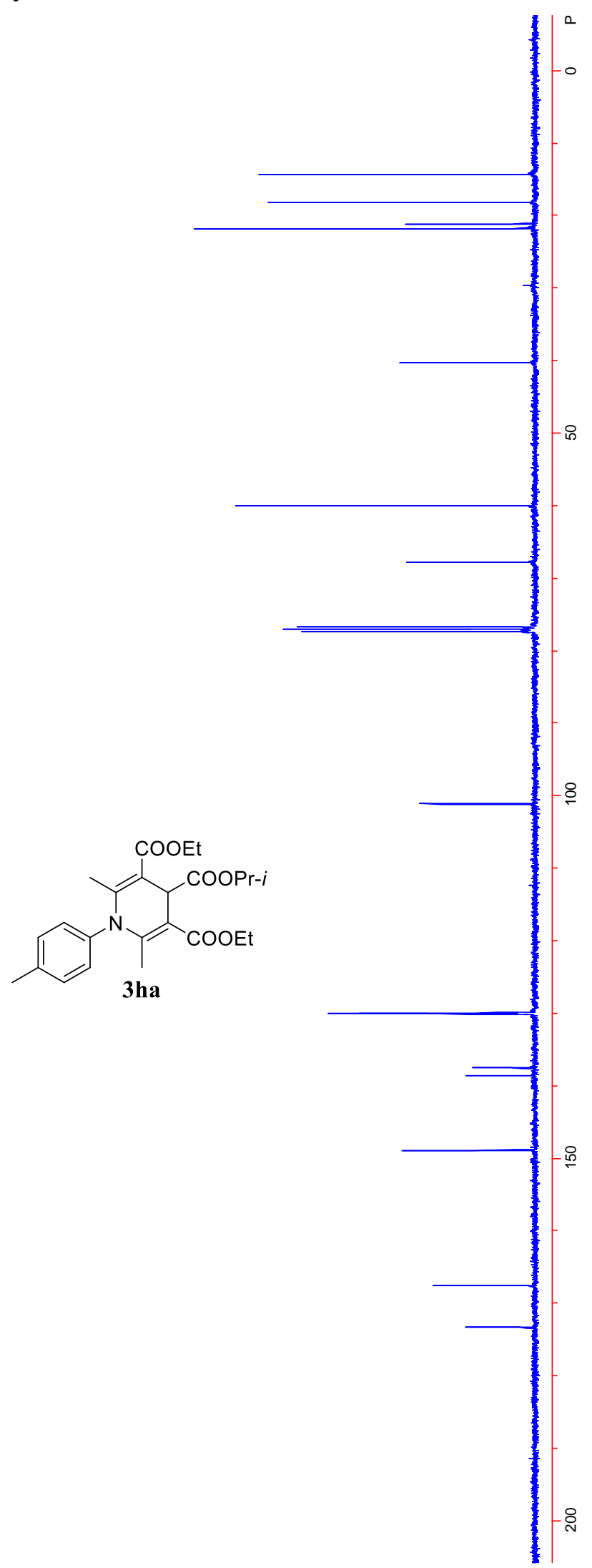

$\downarrow t 0.09$

$68 L \angle 9$

$9 \varepsilon \angle \cdot 9 L \longrightarrow$

$1<\varepsilon^{\circ}<$

$9+เ \vdash \circ$

†96 62 เ

$\varepsilon 80^{\circ} 0 \varepsilon \mathrm{L}$

$\downarrow S S^{\circ} \angle \varepsilon+$

$288^{\circ} 8+1$

$8 \angle 9^{\circ} \angle 91$

0૬ع $\varepsilon \angle เ$ 
HR-MS Spectra of 3,5-diethyl 4-isopropyl 1,4-dihydro-2,6-dimethyl-1-p-tolylpyridine-3,4,5-tricarboxylate 3ha

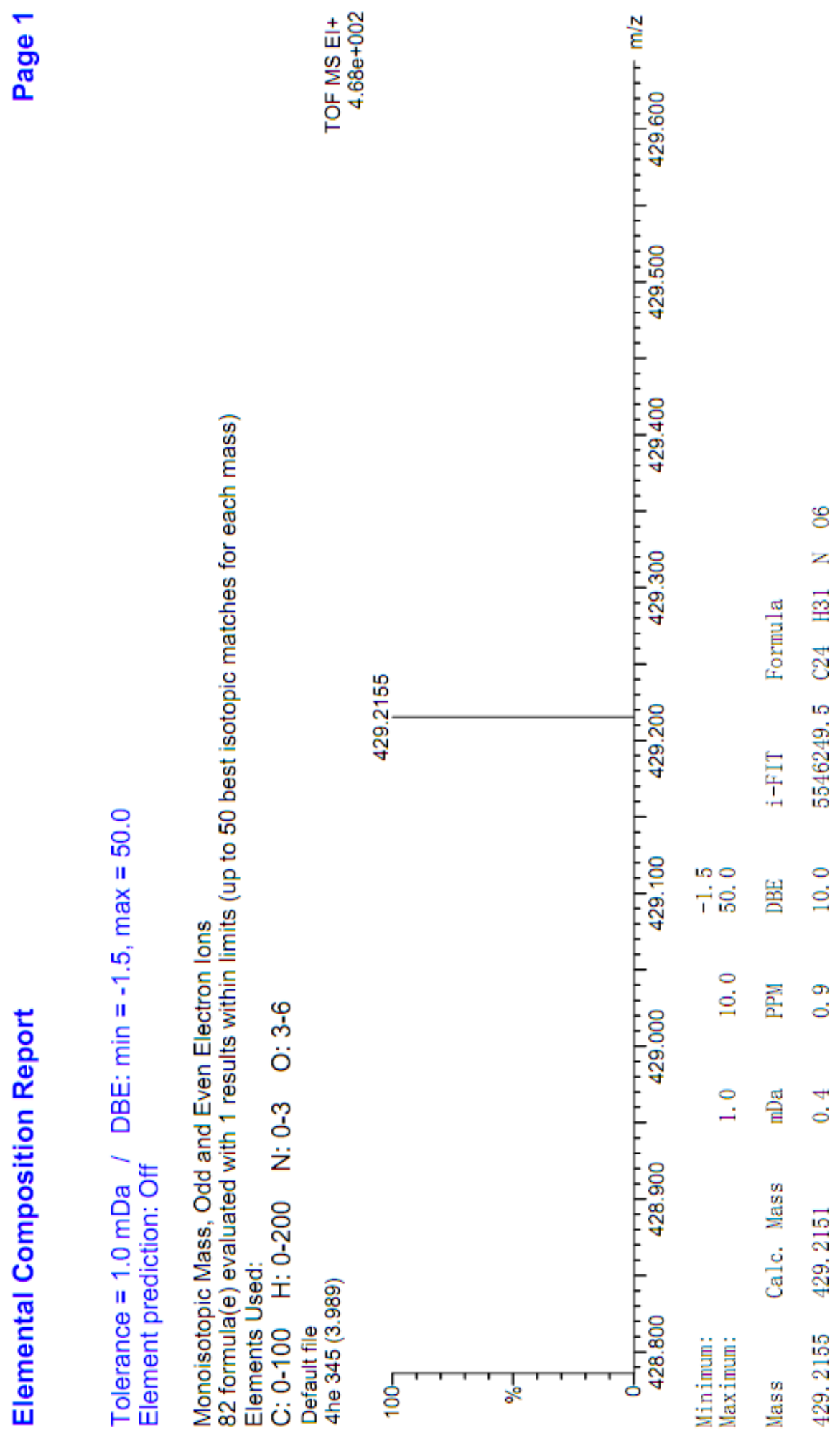


${ }^{1}$ H NMR Spectra of 4-tert-butyl 3,5-diethyl 1,4-dihydro-2,6-dimethyl-1-p-tolylpyridine-3,4,5-tricarbxylate 3ia
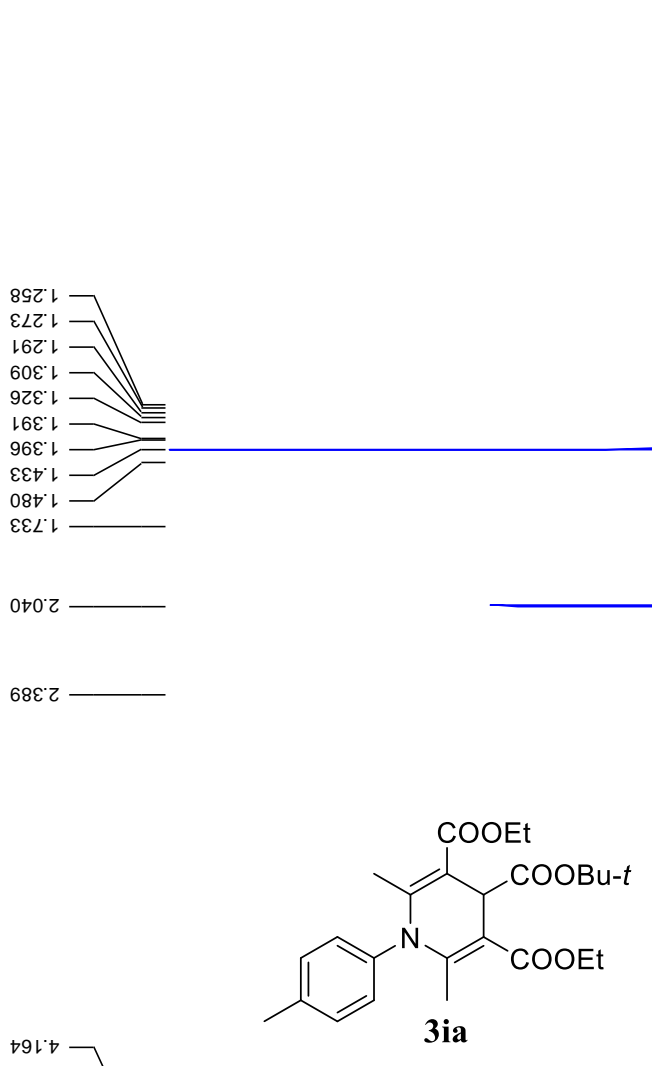

$\forall \angle I^{\circ} \rightarrow$

$28 L^{\circ}$
$16+5$

60ट't

こเで

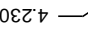

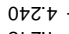

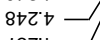

ЦGZ゙†

$\checkmark L L ' D$

S10. $\longrightarrow$

002.1

$002 \div \longrightarrow$

$9 \angle 2<-$

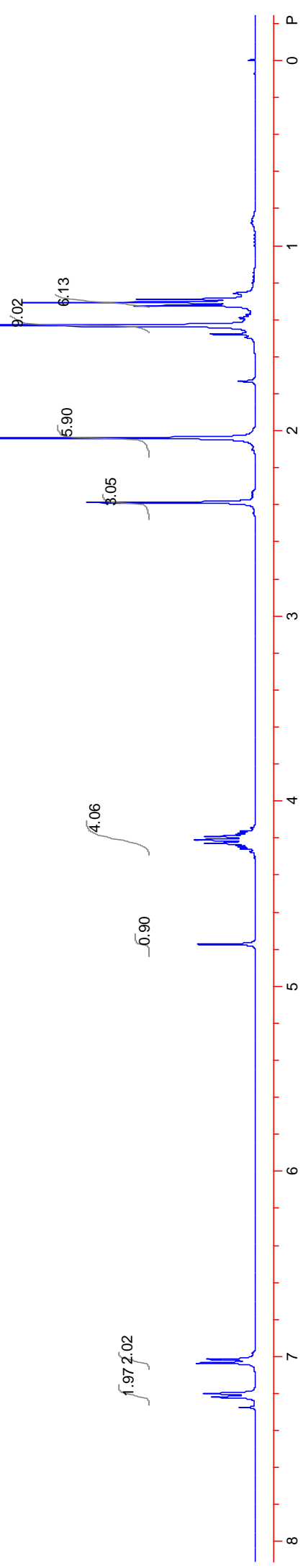


${ }^{13} \mathrm{C}$ NMR Spectra of 4-tert-butyl 3,5-diethyl 1,4-dihydro-2,6-dimethyl-1-p-tolylpyridine-3,4,5-tricarbxylate 3ia

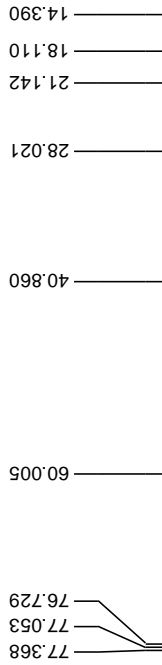

$89 \varepsilon^{\circ} L L$

$9+1.08$

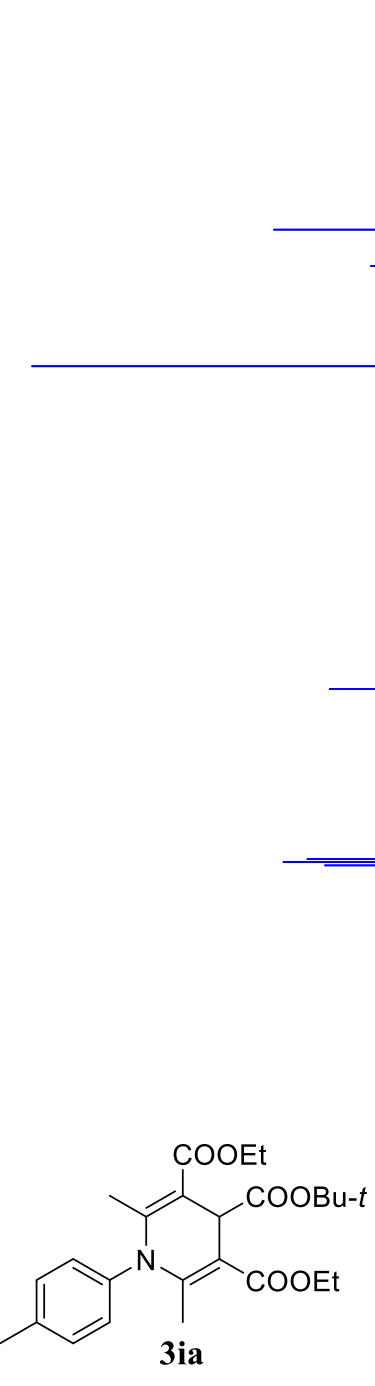

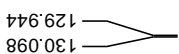

$ヤ เ 9^{\circ} \angle \varepsilon+$

$9 \angle G^{\circ} 8 \varepsilon+\longrightarrow$

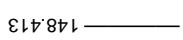

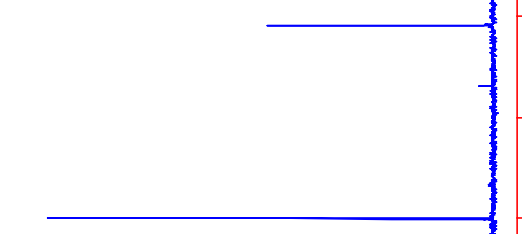

$9 \angle t+101$

$9 Z \angle \angle 29$

$608: 2<1$

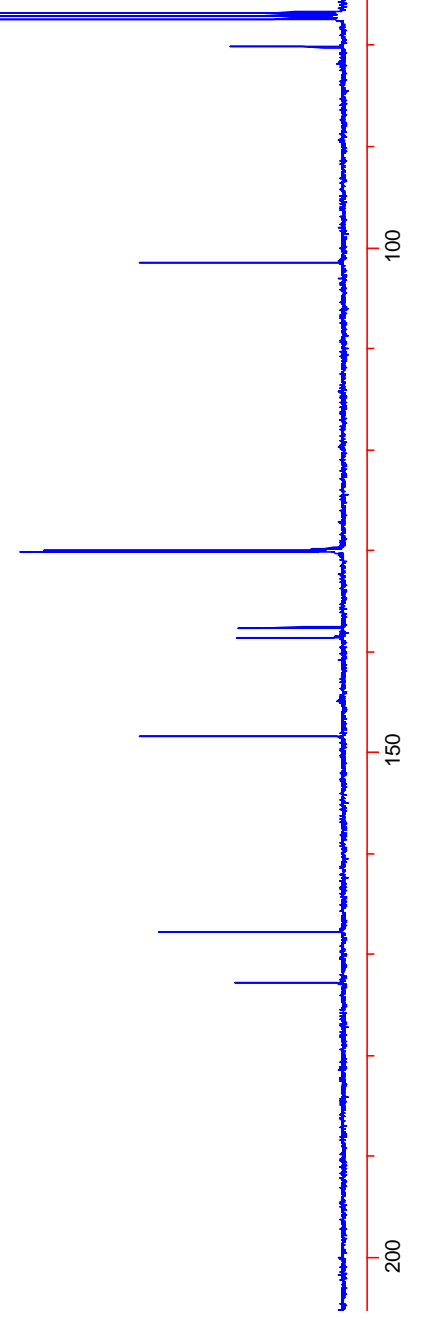


HR-MS Spectra of 4-tert-butyl 3,5-diethyl 1,4-dihydro-2,6-dimethyl-1-p-tolylpyridine-3,4,5-tricarbxylate 3ia

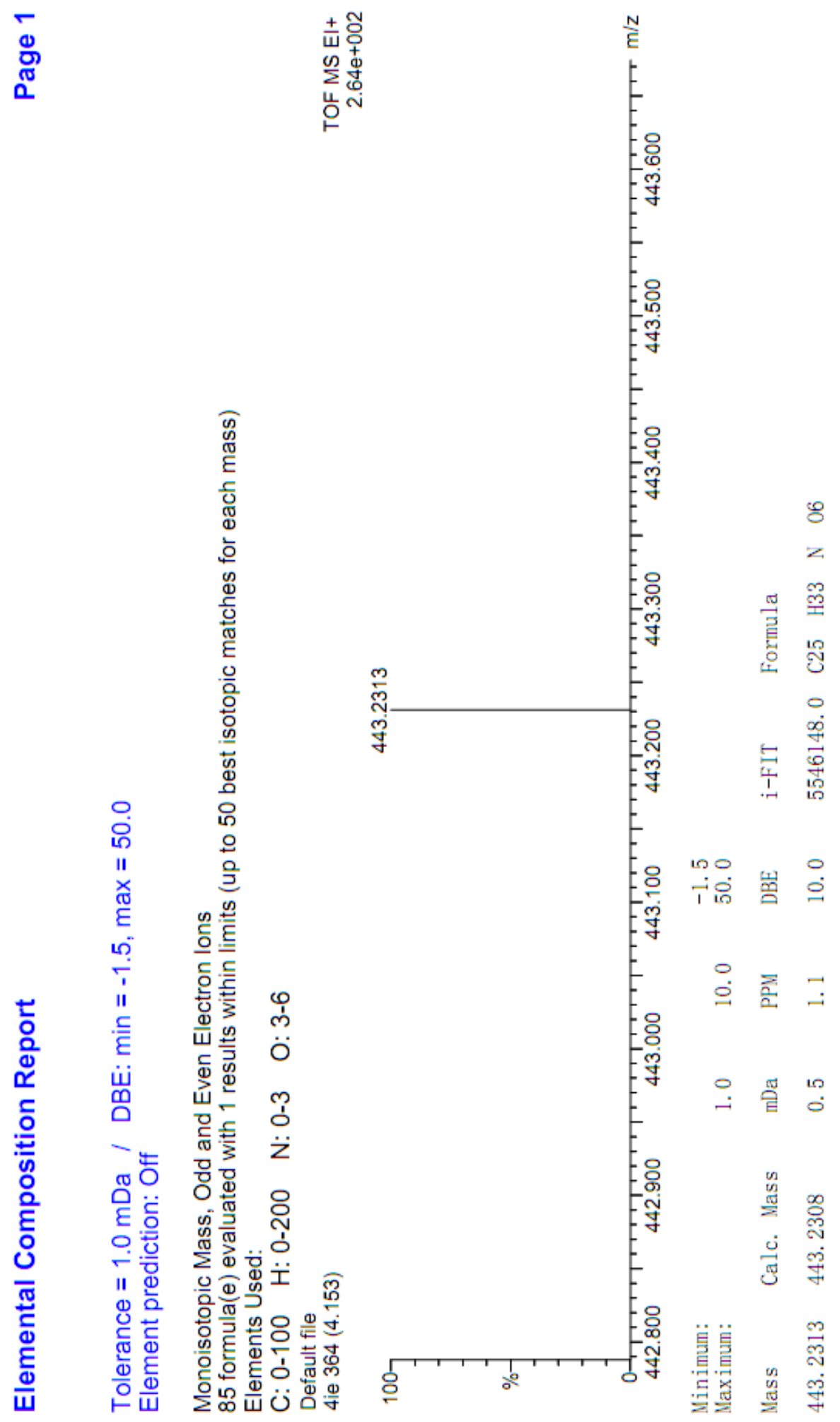


${ }^{1} \mathrm{H}$ NMR Spectra of 4-benzyl 3,5-diethyl 1,4-dihydro-2,6-dimethyl-1-p-tolylpyridine3,4,5-tricarboxylate $\mathbf{3 j a}$

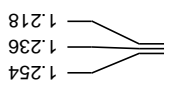

$0+0 \cdot 2$

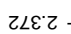

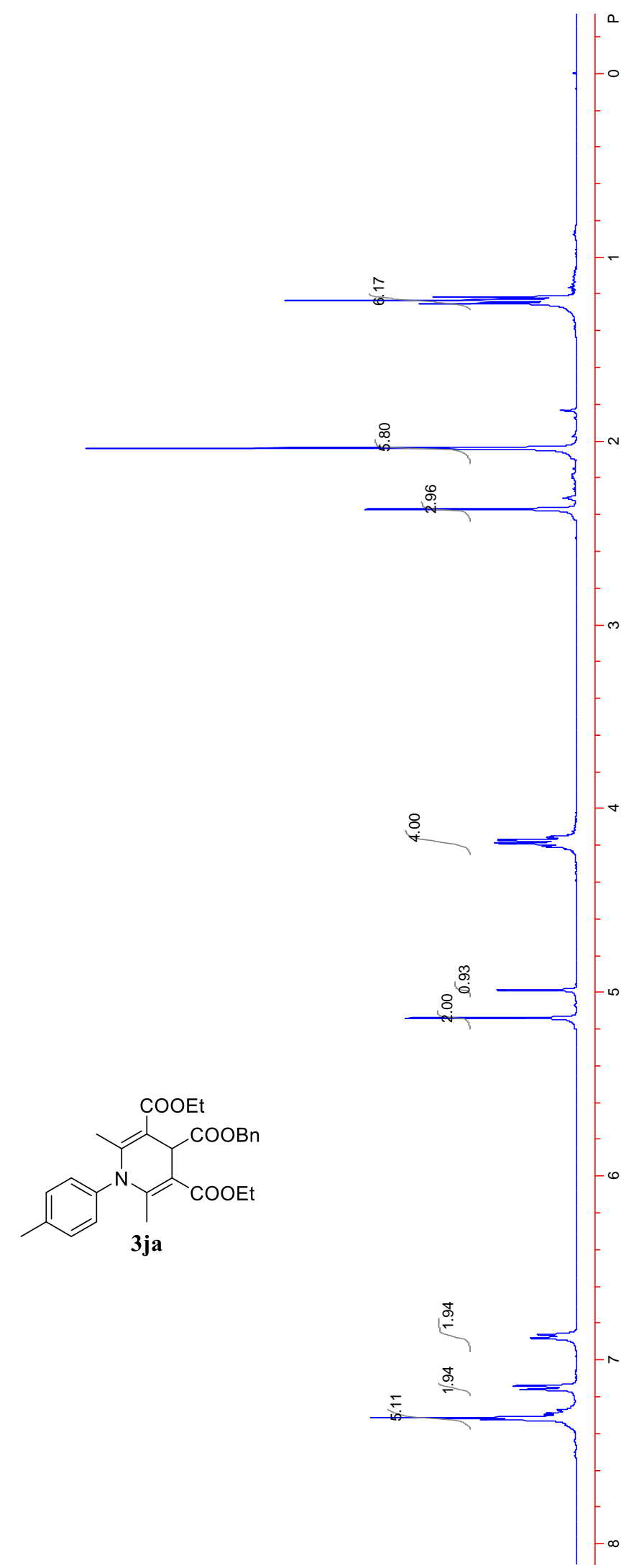

¿s.'t

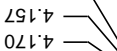

sLt.

$881^{\circ} t$

$\varepsilon 61^{\circ} \mathrm{t}$

SOZ'

$886^{\circ} t$

$0+1 \cdot 9$

เ. 
${ }^{13} \mathrm{C}$ NMR Spectra of 4-benzyl 3,5-diethyl 1,4-dihydro-2,6-dimethyl-1-p-tolylpyridine3,4,5-tricarboxylate $\mathbf{3 j a}$

๕8ごเト

$99 \cdot 81$

$\angle \varepsilon+\cdot L$

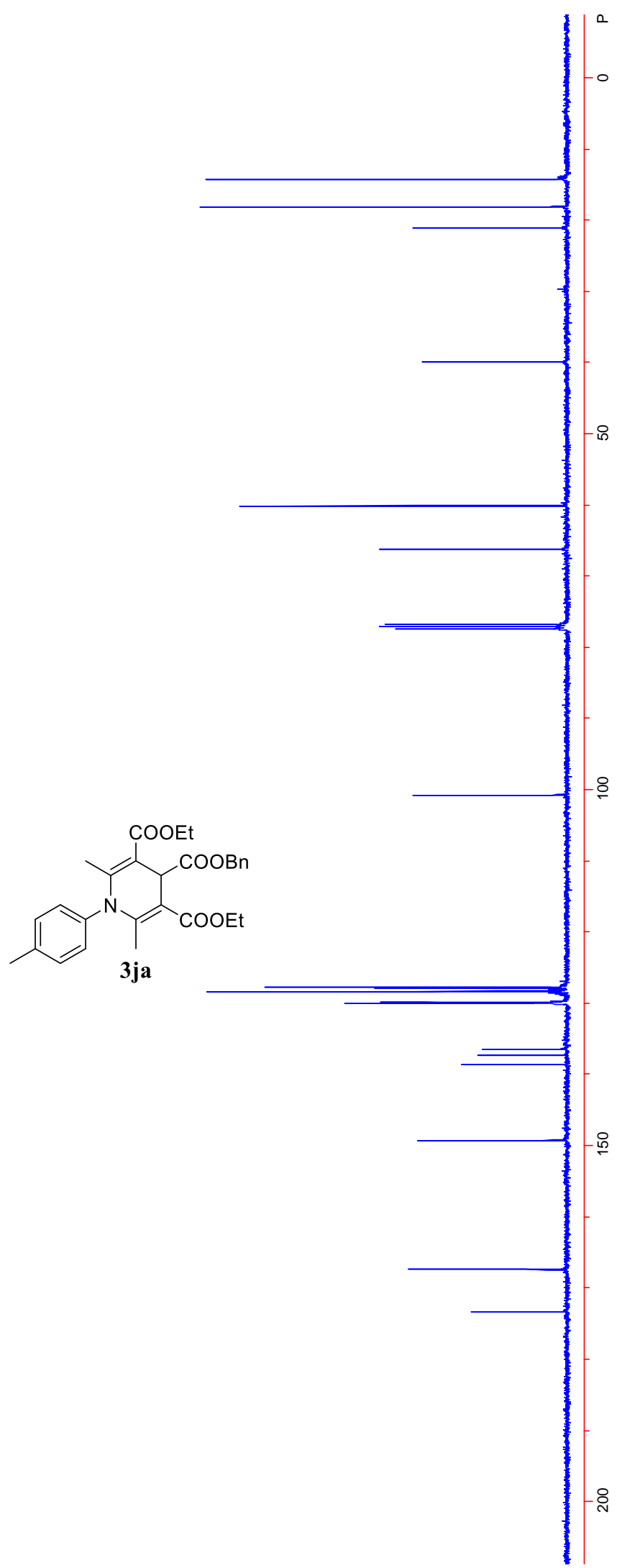

$896.6 \varepsilon$

$19 L^{\circ} 001$

$\angle L L \angle Z 1$

$168.2 Z 1 \longrightarrow$

वहع 8टा

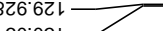

$\angle t^{\circ} 9 \varepsilon+$

$\mathrm{s} 6 \mathrm{C} \angle \varepsilon+\longrightarrow$

st9. $8 \varepsilon+$

$1276+$

LZナ $\angle 9 L$

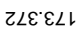


HR-MS Spectra of 4-benzyl 3,5-diethyl 1,4-dihydro-2,6-dimethyl-1-p-tolylpyridine3,4,5-tricarboxylate $3 \mathbf{j a}$

ฮ্ণ
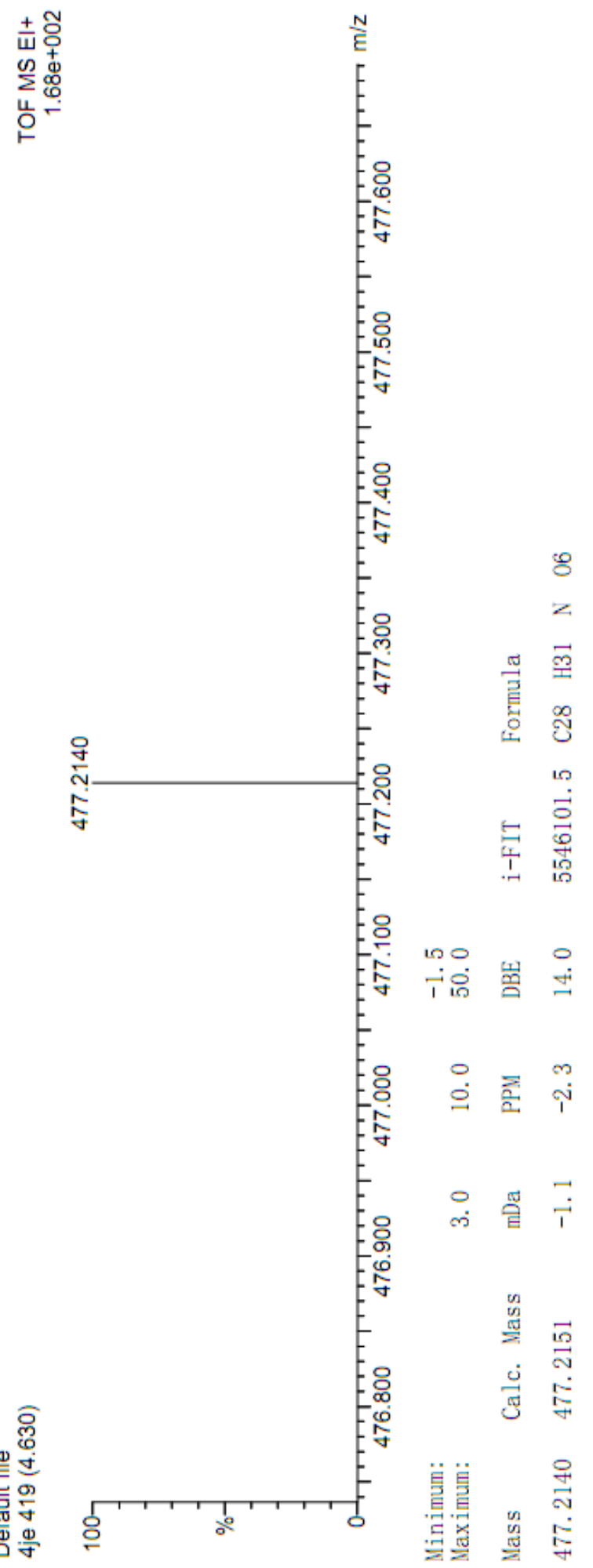
${ }^{1}$ H NMR Spectra of 4-ethyl 3,5-dimethyl 1,4-dihydro-1-(4-methoxyphenyl)-2,6-dimethylpyridine-3,4,5-tricarboxylate $\mathbf{3 b b}$
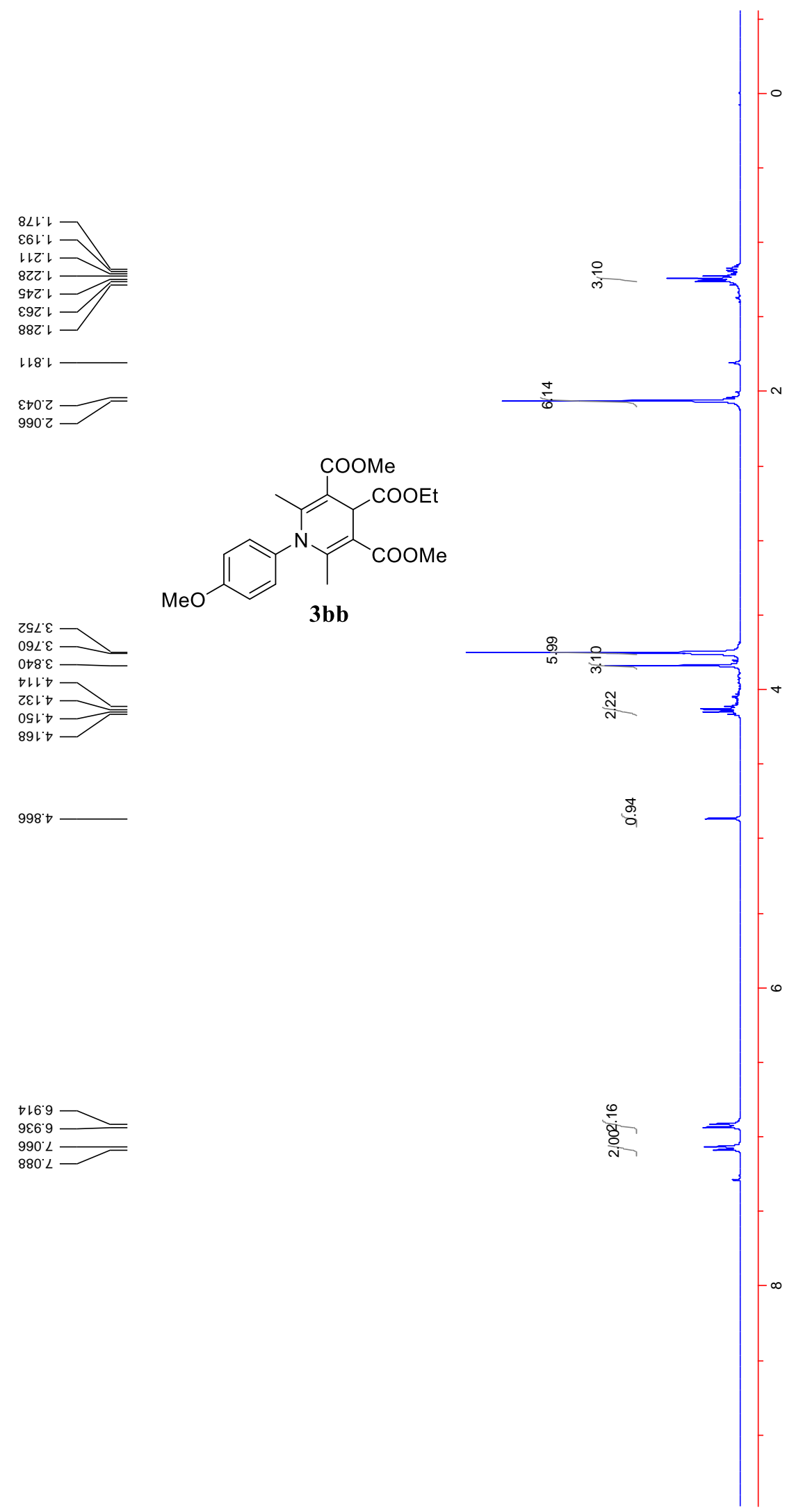

$+16.9 \square$
$986^{\circ} 9 \square$
$990^{\circ} \mathrm{L} \longrightarrow$
$880^{\circ} \mathrm{L}$

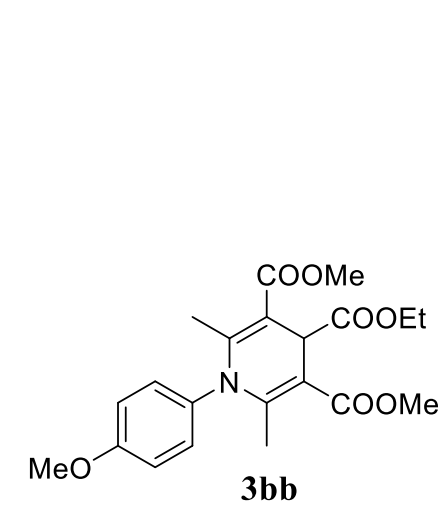

$0+8 \cdot \varepsilon$

七トเ't

टहเ'

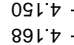

998 ॰

$\mathbf{3 b b}$ 
${ }^{1} \mathrm{H}$ NMR Spectra of 4-ethyl 3,5-diisopropyl 1,4-dihydro-1-(4-methoxyphenyl)-2,6dimethylpyridine-3,4,5-tricarboxylate 3bc
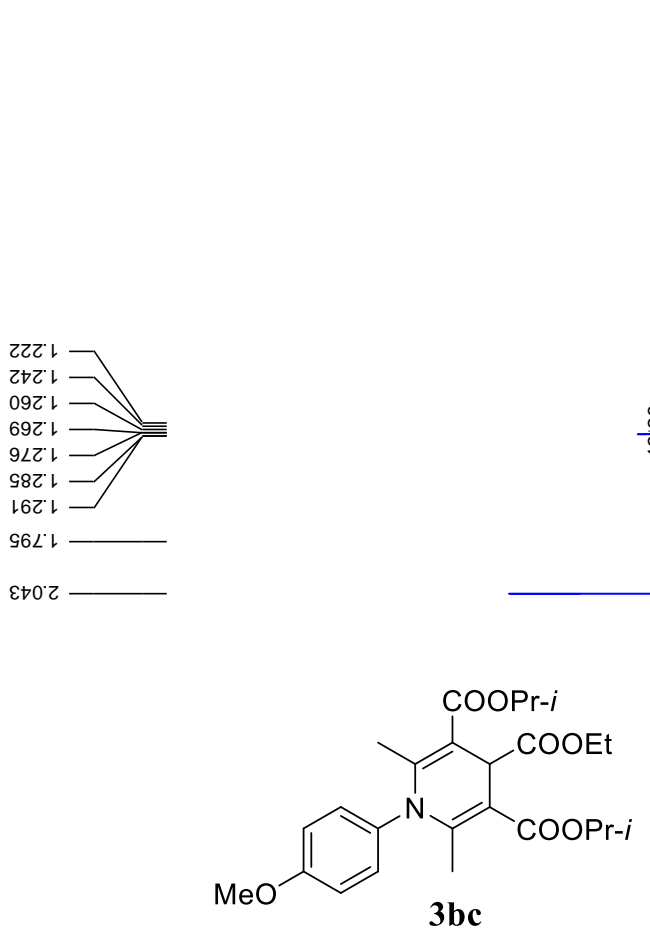

५๕8. $\mathcal{-}$

मレ't

$62+5$

เ91.

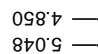

$790^{\circ}$

$080 \circ$

$960^{\circ} \mathrm{S}$

1.1.9

$8069-$

เ乙6. 9 -

$6 \angle 0 L \square$
$10 L^{\circ} \angle-$

$3 b c$
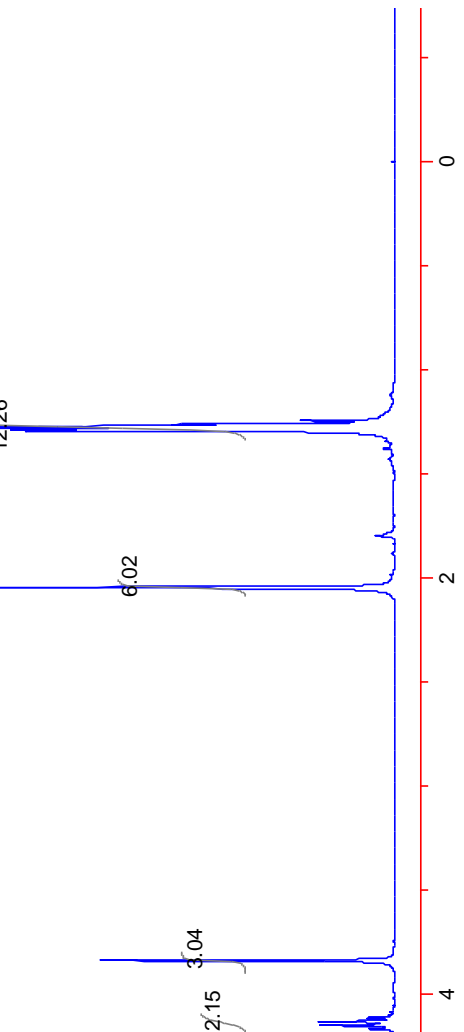

응

总

$28 \mathrm{Z}^{\circ} \mathrm{L}$

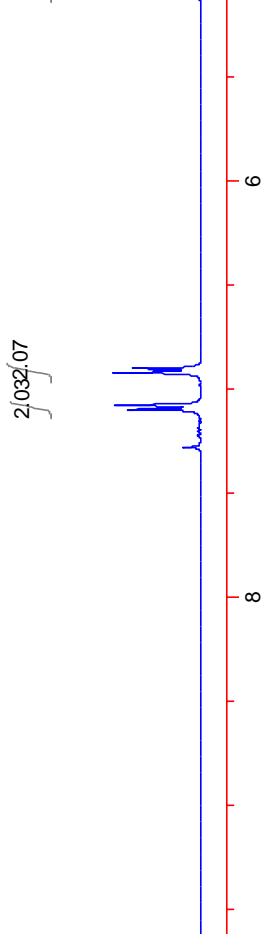


${ }^{1} \mathrm{H}$ NMR Spectra of 3,5-di-tert-butyl 4-ethyl 1,4-dihydro-1-(4-methoxyphenyl)-2,6dimethylpyridine-3,4,5-tricarboxylate $3 \mathbf{b d}$
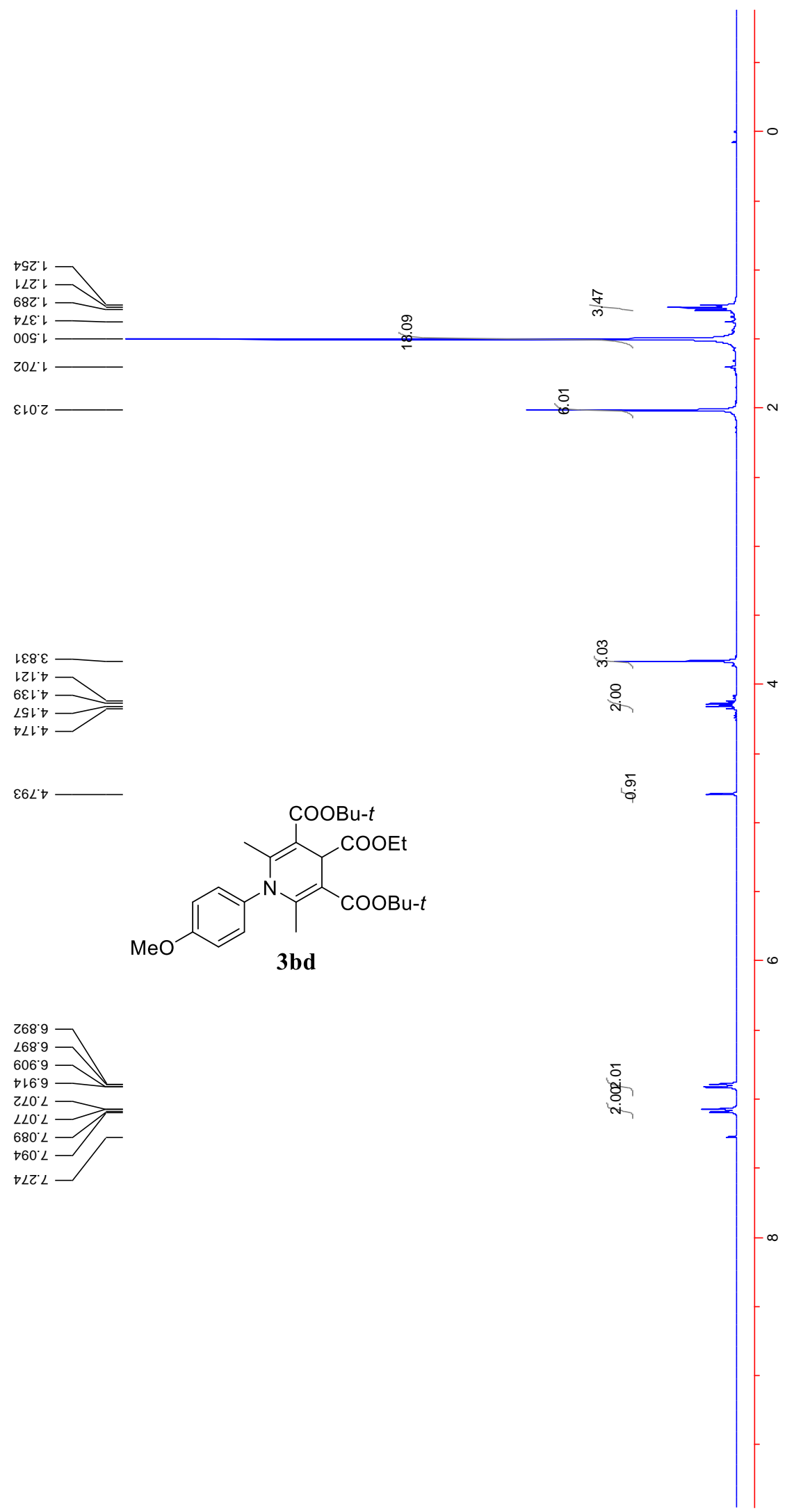
${ }^{1}$ H NMR Spectra of ethyl 3,5-diacetyl-1,4-dihydro-1-(4-methoxyphenyl)-2,6-dimethylpyridine-4-carboxylate $3 \mathbf{b e}$
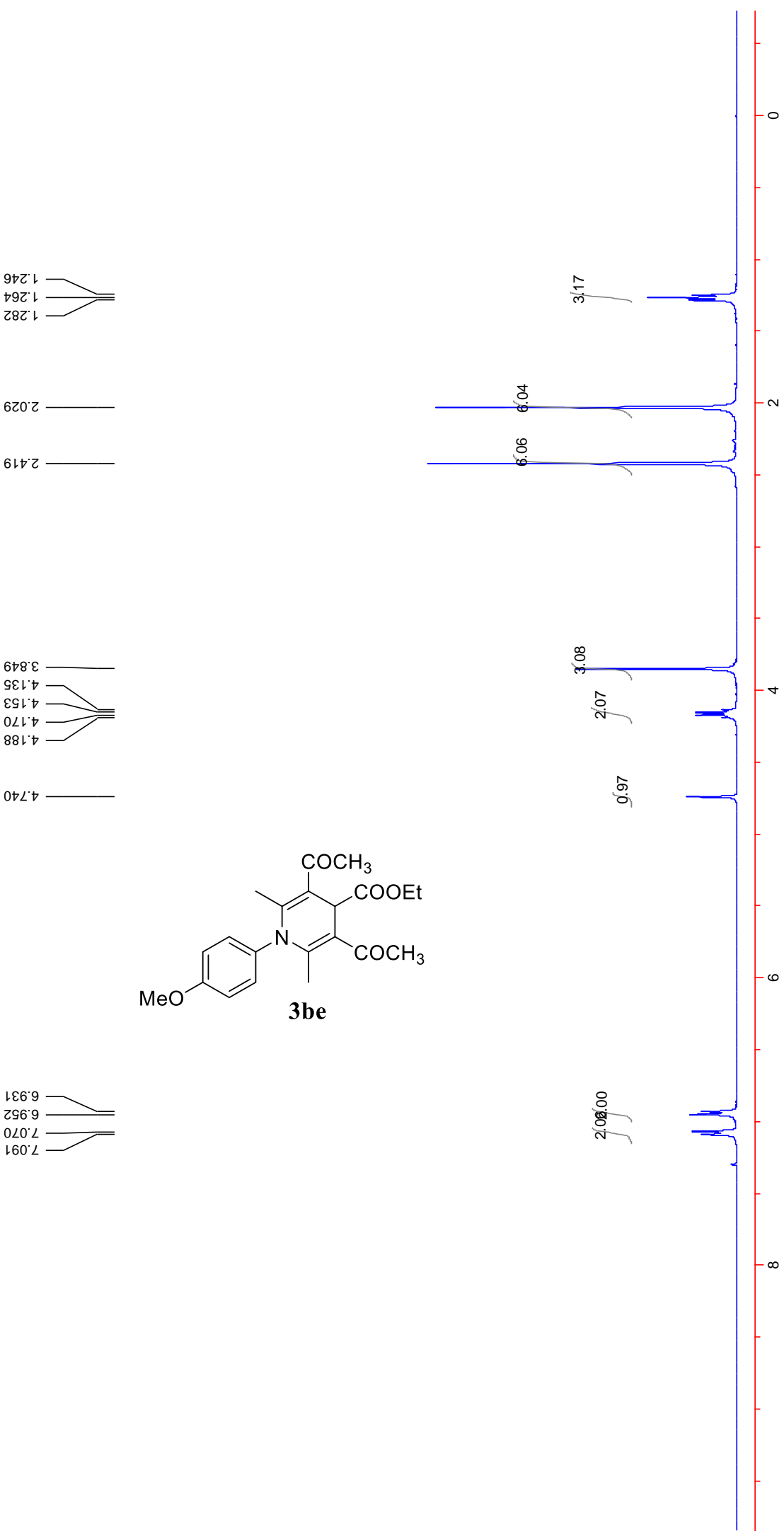

$186^{\circ} 9 \square$
$2966^{\circ}$
$0 \angle 0 \circ \square$

sEเ

$\varepsilon 9+\circ \longrightarrow$

$0 \triangleright L \cdot t$<smiles>CCOC(=O)C1=C(C)N(c2ccc(OC)cc2)C(C)=C(C(C)=O)C1C(=O)OCC</smiles>

3 be 
${ }^{1}$ H NMR Spectra of ethyl 3,5-dibenzoyl-1-(4-methoxyphenyl)-2,6-dimethyl-1,4-dihydropyridine-4-carboxylate $\mathbf{3 b f}$

$000^{\circ} 0^{-}$

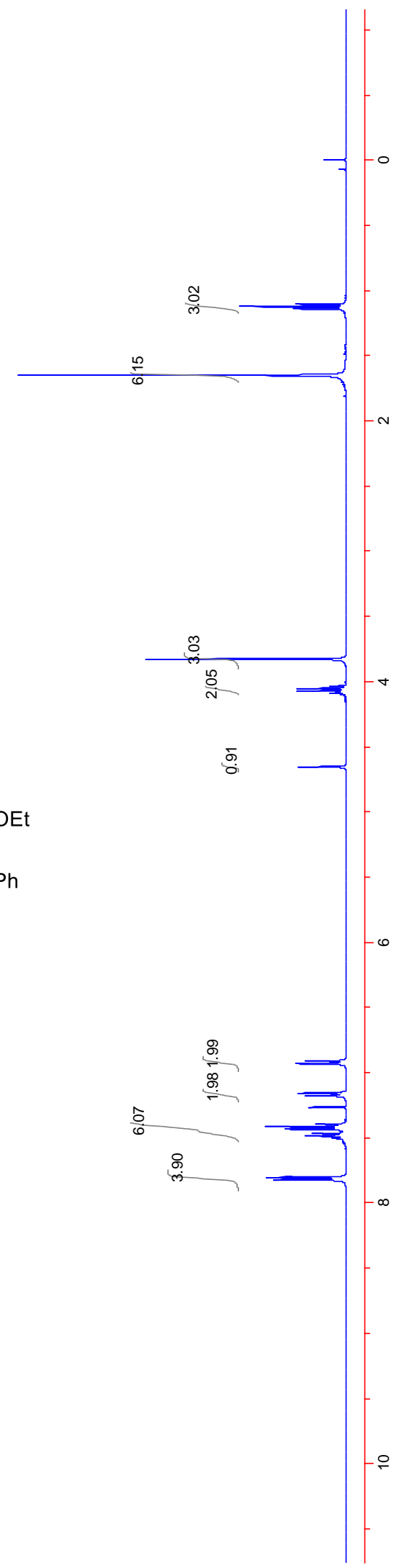

$\angle O L \cdot$

SCL.

เ.9. เ
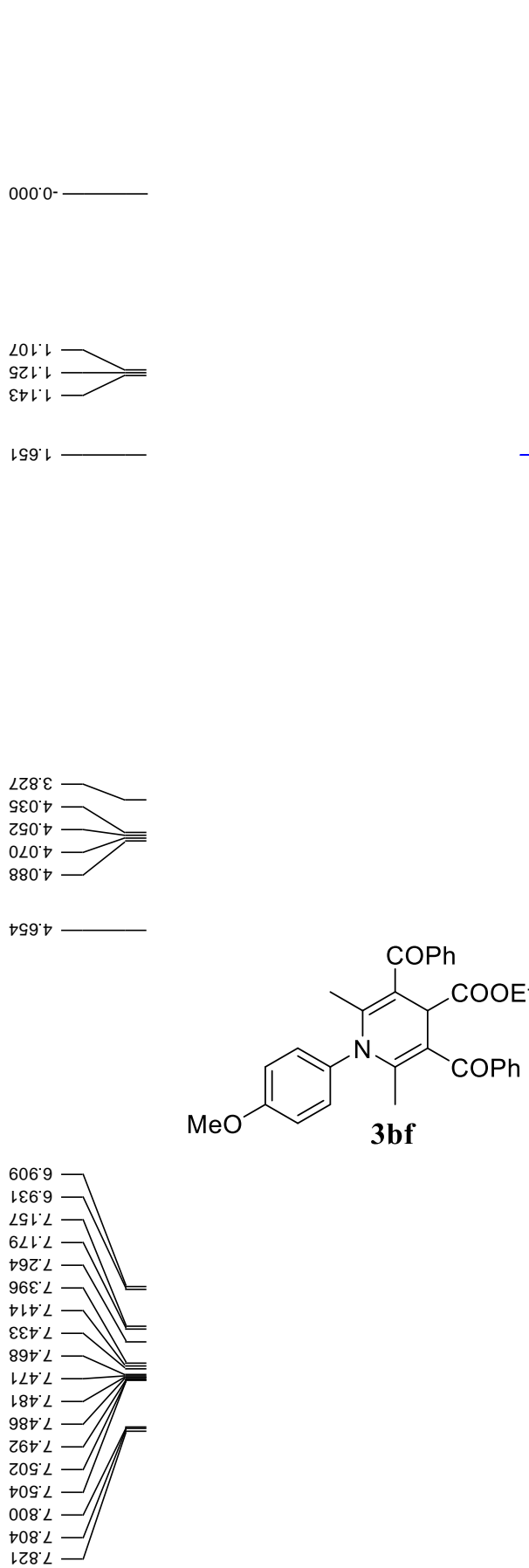

t99 ‘ 
${ }^{1} \mathrm{H}$ NMR Spectra of diethyl 5-acetyl-1,4-dihydro-2,6-dimethyl-1-p-tolylpyridine-3,4dicarboxylate 3aae
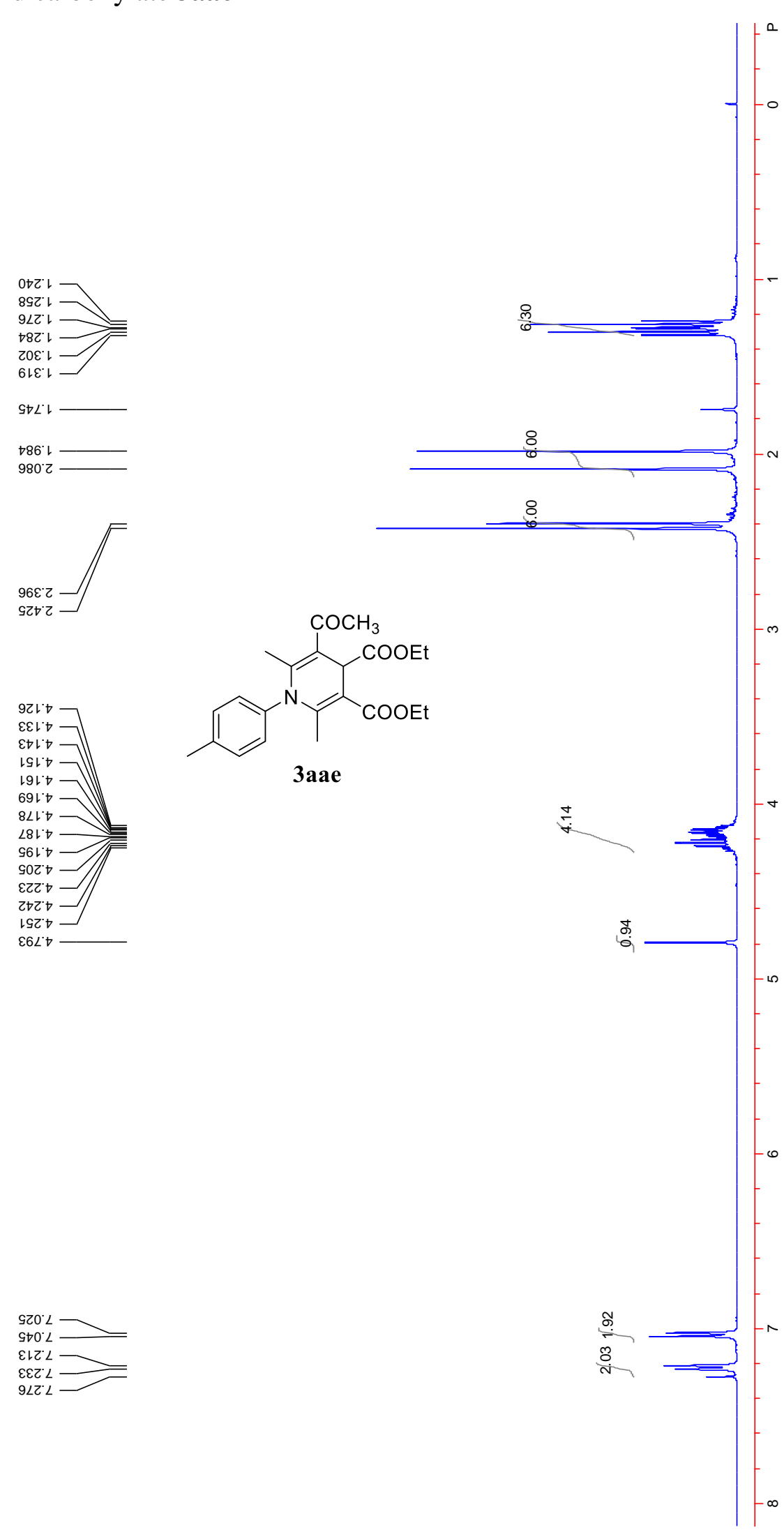
${ }^{13} \mathrm{C}$ NMR Spectra of diethyl 5-acetyl-1,4-dihydro-2,6-dimethyl-1-p-tolylpyridine-3,4dicarboxylate 3aae

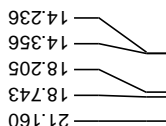

$09+12$

$8+8 \cdot 62$

$298^{\circ} 0 t$<smiles></smiles>

$\downarrow \angle 66^{\circ} 621$

$\angle 20^{\circ} 0 \varepsilon+\longrightarrow$

$\varepsilon \angle Z \subset \angle L$
$2 เ 8 \cdot 8 \varepsilon+$

$0 \varsigma 8 \angle \forall \vdash$

ZLt 6 十

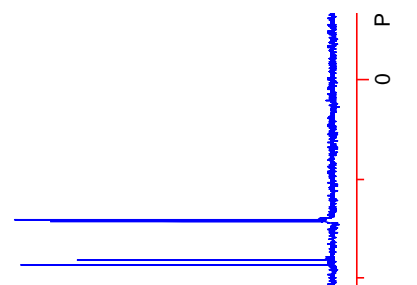

$9 \triangleright 2 \cdot 09$

$80609 \square$

$\angle Z L \cdot 9 L$

$\nabla S 0^{\circ} \angle L$
$0 \angle \varepsilon^{\circ} \angle L$

966.001

요

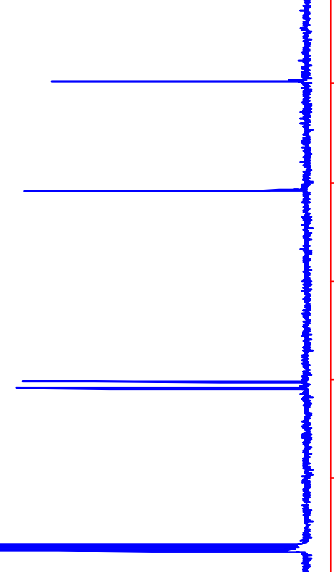

عटะ 60 เ

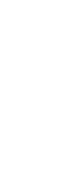

$892 \angle 91$

$\dashv 6{ }^{*} \varepsilon\llcorner\llcorner$

206.861

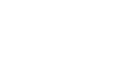


HR-MS Spectra of diethyl 5-acetyl-1,4-dihydro-2,6-dimethyl-1-p-tolylpyridine-3,4dicarboxylate 3aae

홈
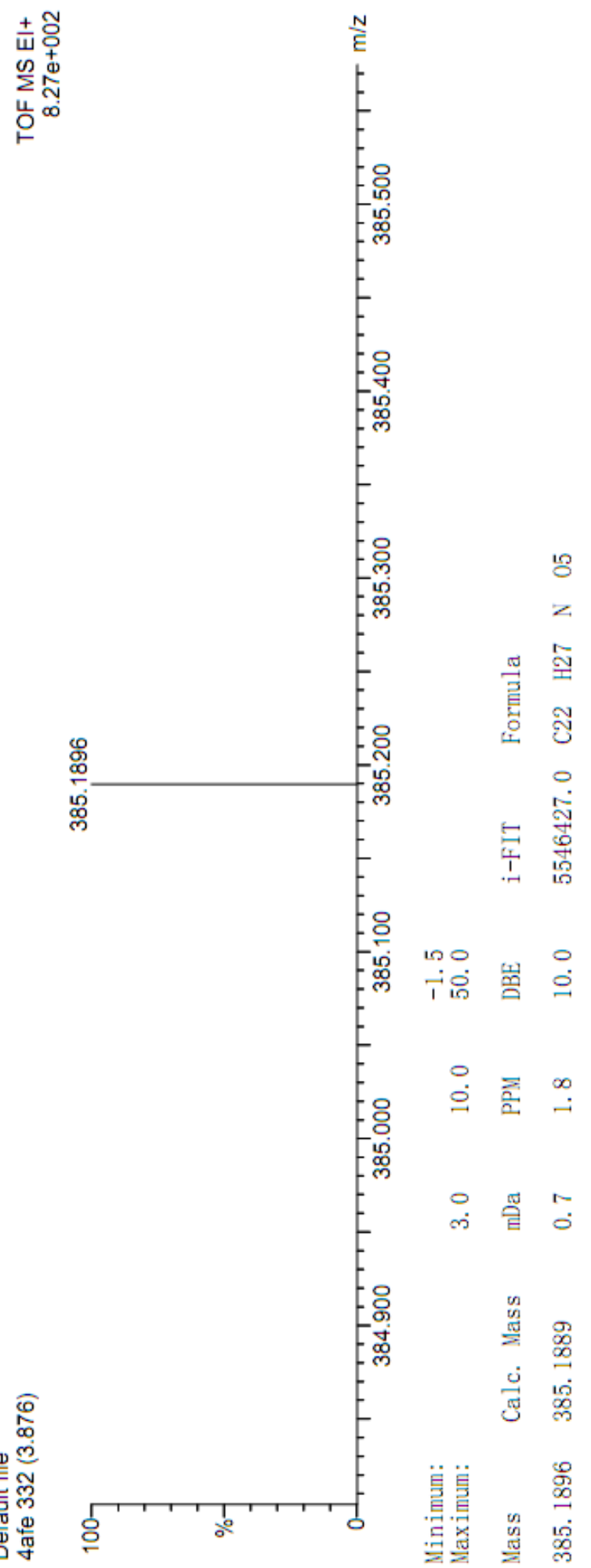
${ }^{1}$ H NMR Spectra of diethyl 5-acetyl-1,4-dihydro-1-(4-methoxyphenyl)-2,6-dimethylpyridine-3,4-dicarboxylate 3bae
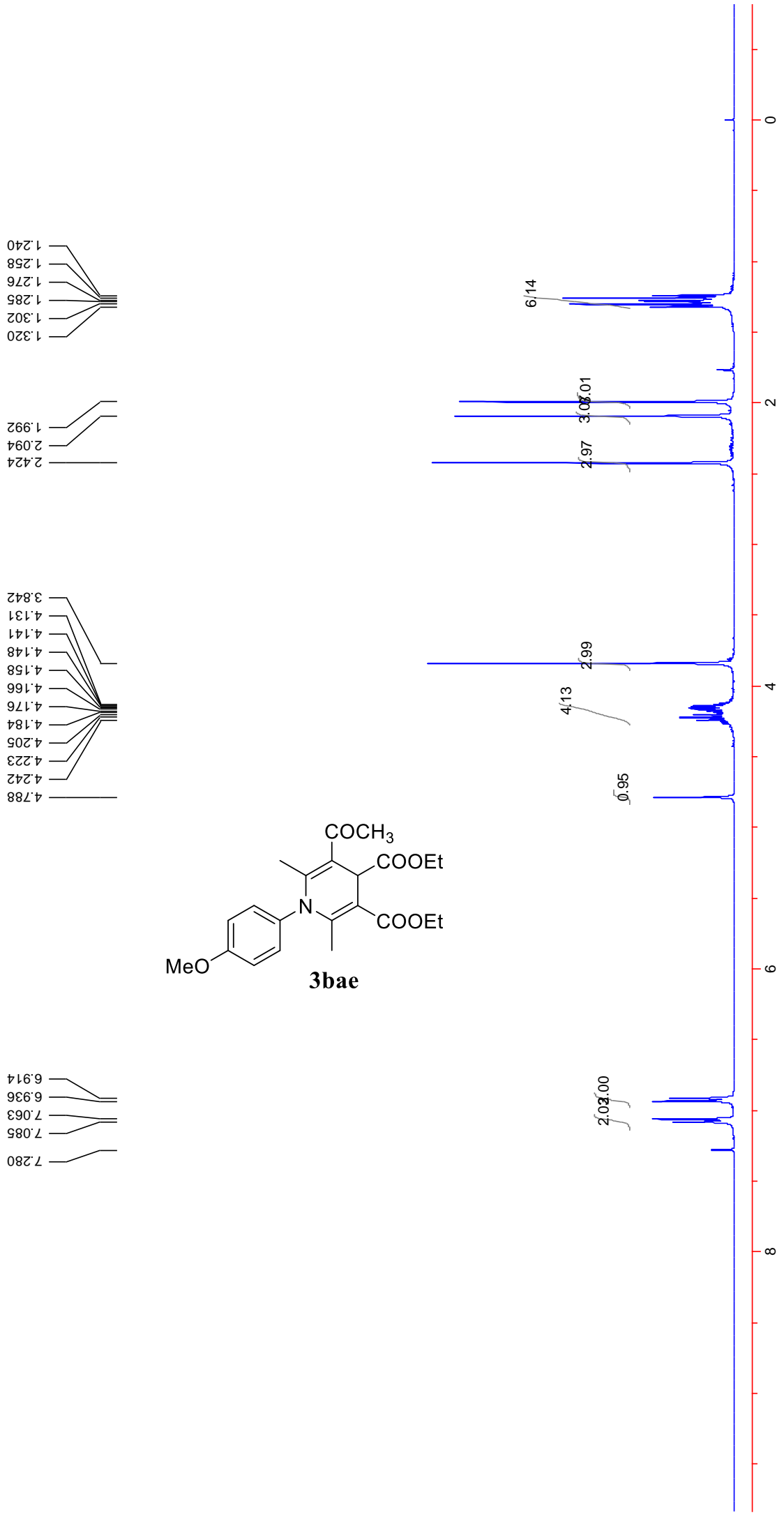

เเ6.9

$\varepsilon 90^{\circ} \mathrm{L} \longrightarrow$
$\square$

082. 
${ }^{1}$ H NMR Spectra of ethyl 4,5-diacetyl-1,4-dihydro-2,6-dimethyl-1-p-tolylpyridine-3carboxylate 3gae
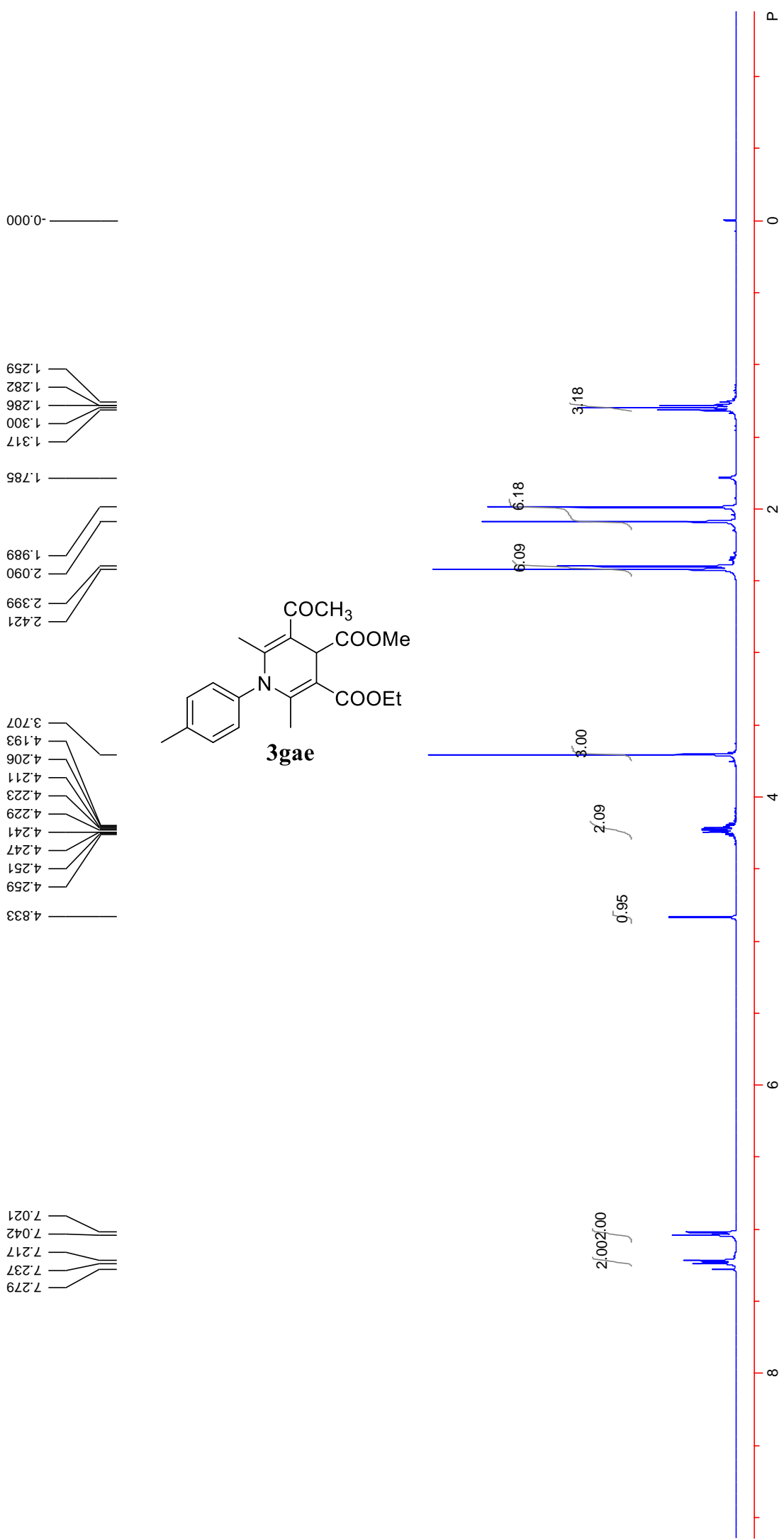
${ }^{13} \mathrm{C}$ NMR Spectra of ethyl 4,5-diacetyl-1,4-dihydro-2,6-dimethyl-1- $p$-tolylpyridine-3carboxylate 3gae

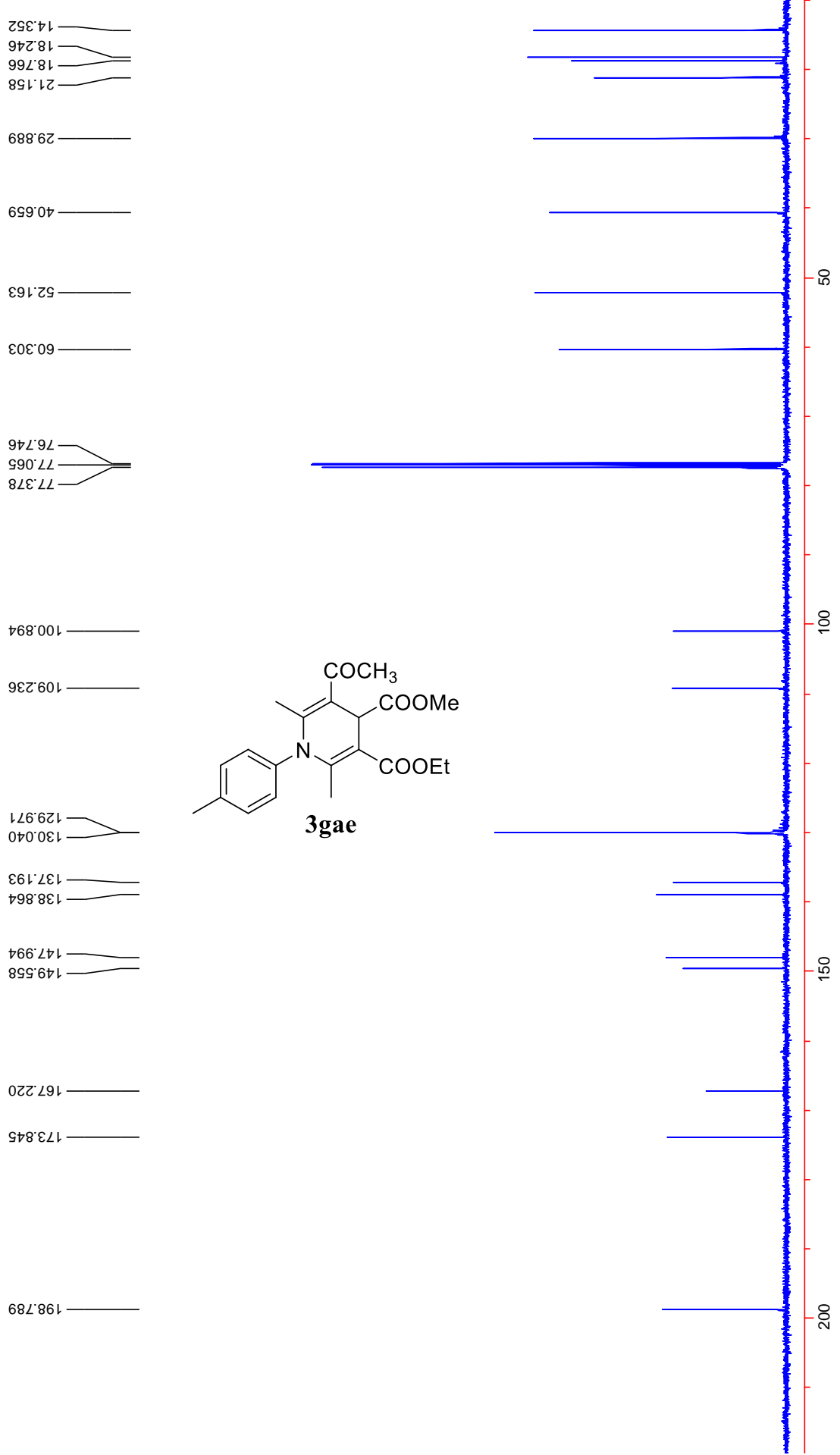


HR-MS Spectra of ethyl 4,5-diacetyl-1,4-dihydro-2,6-dimethyl-1-p-tolylpyridine-3carboxylate 3gae

ণ্ঠ

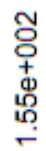
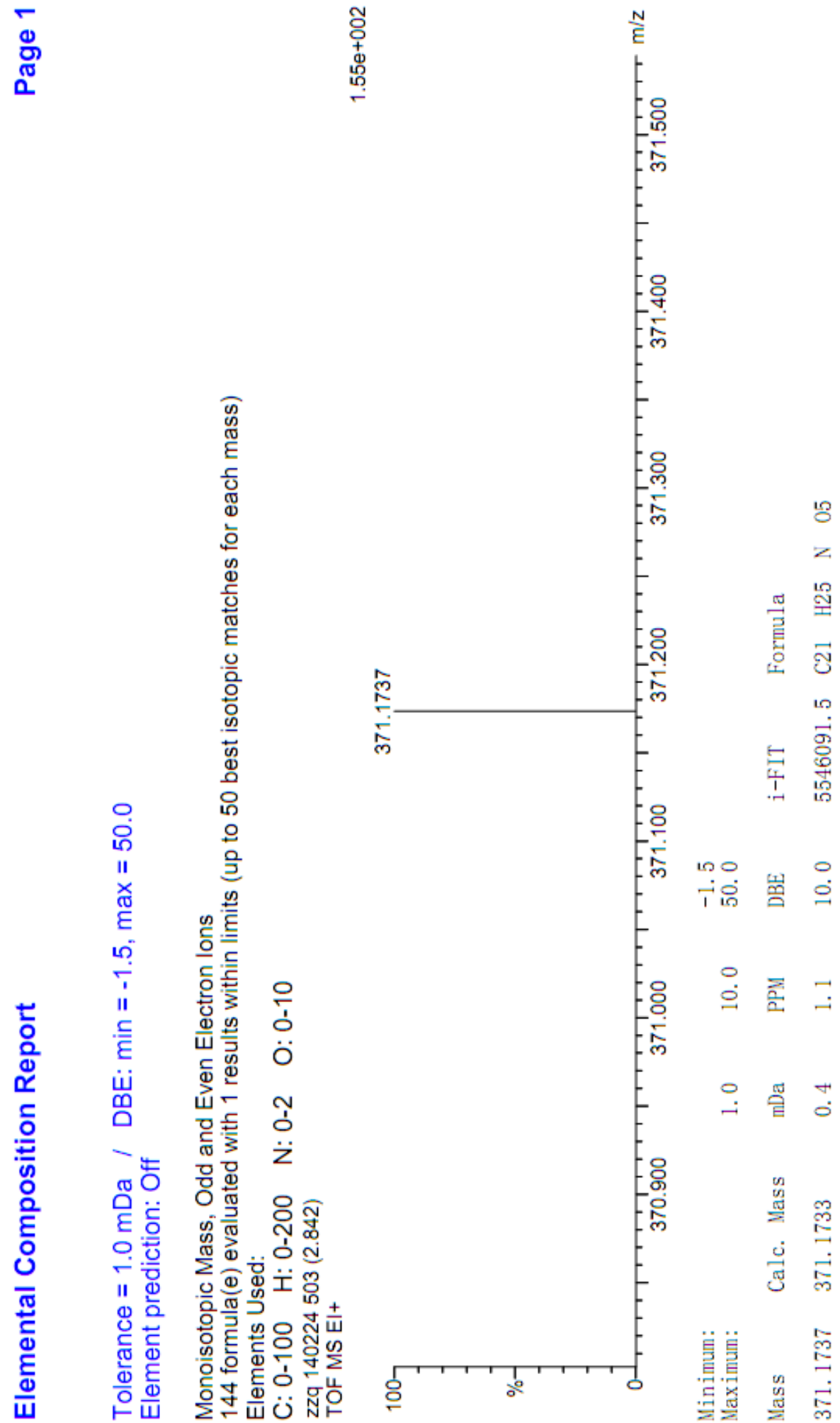
${ }^{1}$ H NMR Spectra of 4-ethyl 3-methyl 5-acetyl-1,4-dihydro-1-(4-methoxyphenyl)-2,6dimethylpyridine-3,4-dicarboxylate $3 \mathbf{b b e}$
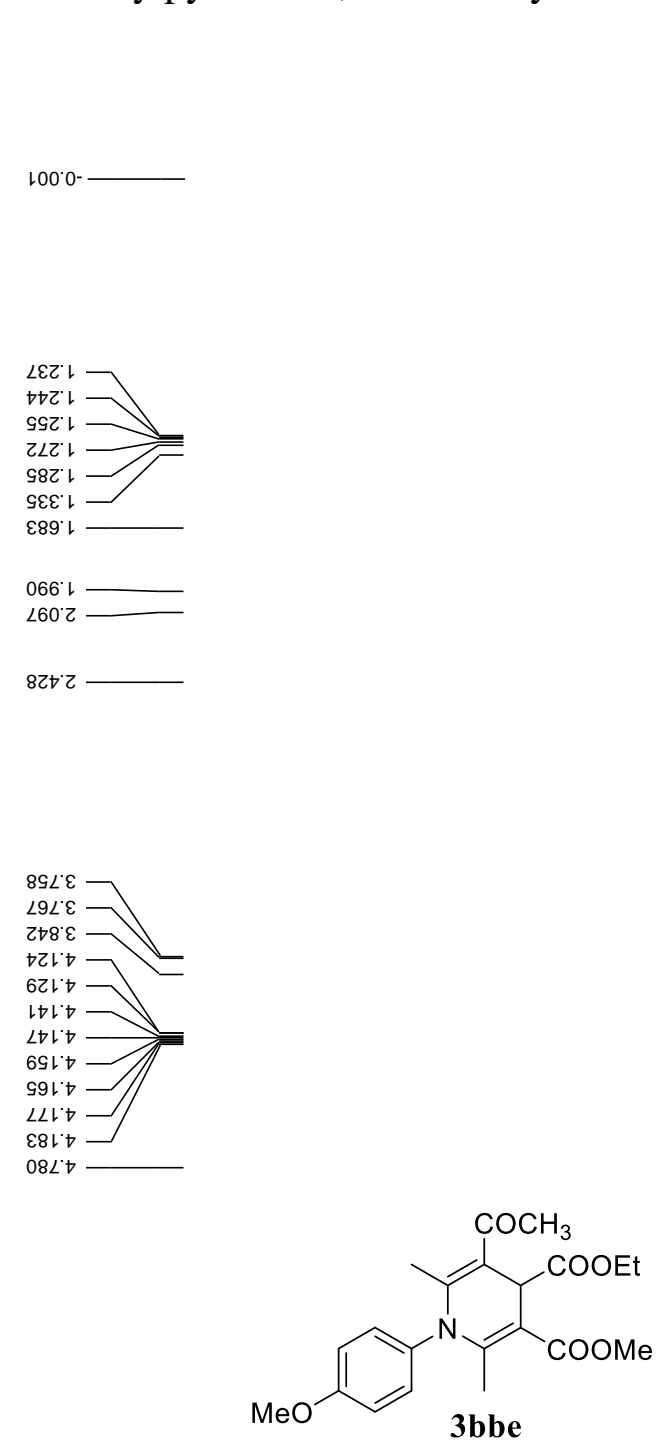

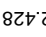

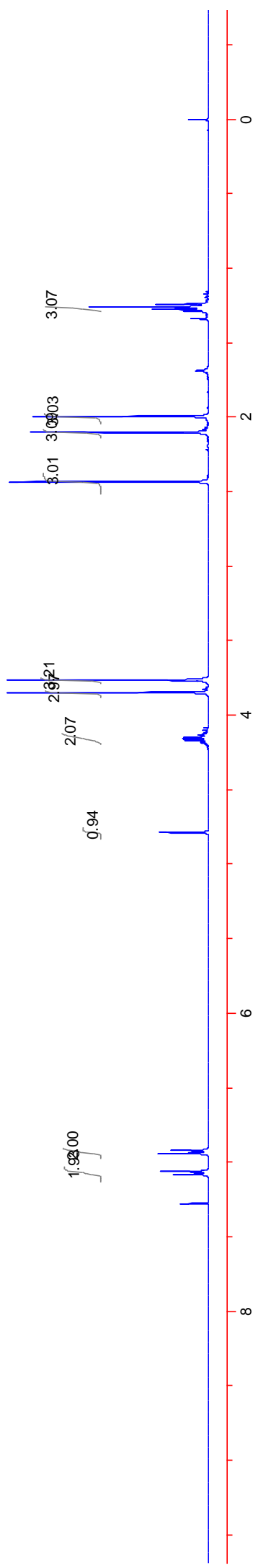

เเ6.9

$986^{\circ} 9$

SSO $\angle \longrightarrow$

$\angle L O L$

$1 L Z \subset \subset$ 
${ }^{1}$ H NMR Spectra of 4-ethyl 3-isopropyl 5-acetyl-1,4-dihydro-1-(4-methoxyphenyl)2,6-dimethylpyridine-3,4-dicarboxylate 3bce

$000^{\circ} 0^{-}$
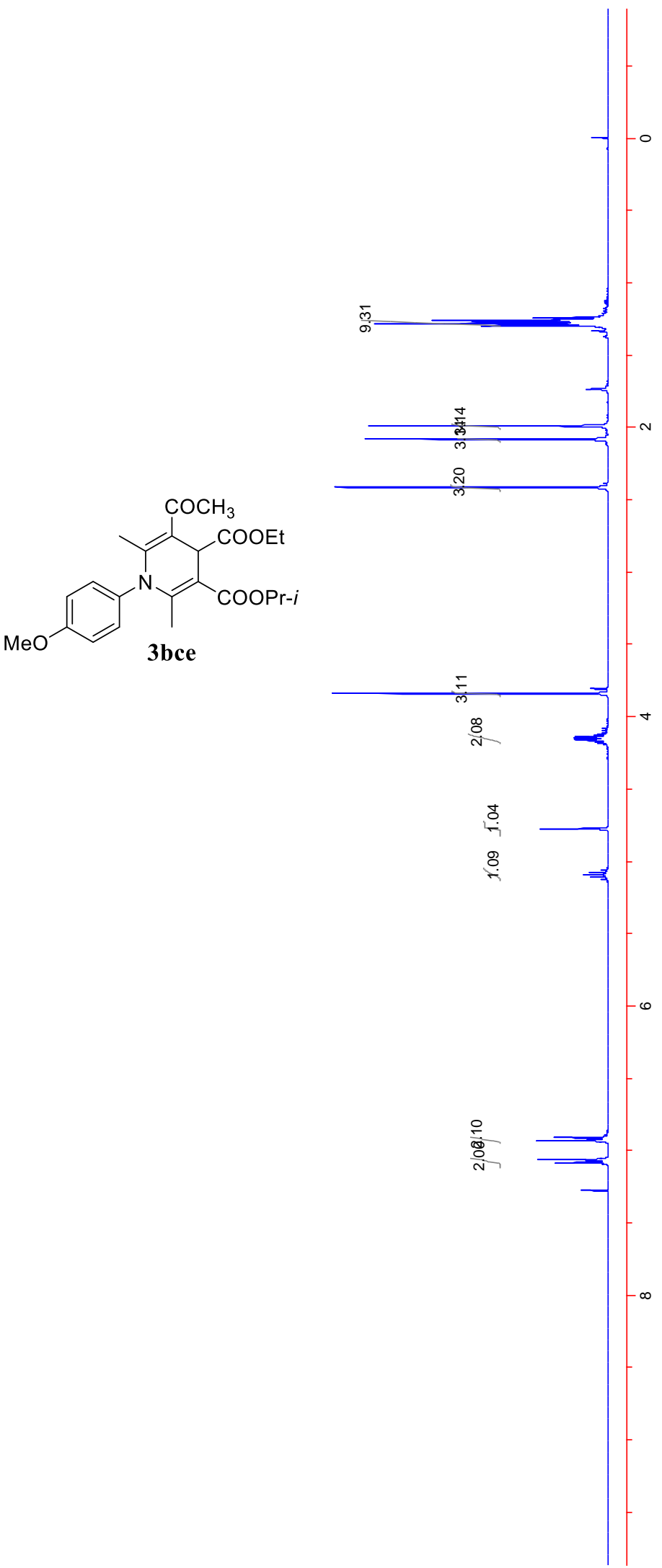
${ }^{1}$ H NMR Spectra of 3-tert-butyl 4-ethyl 5-acetyl-1,4-dihydro-1-(4-methoxyphenyl)2,6-dimethylpyridine-3,4-dicarboxylate 3bde

$0000^{\circ}$

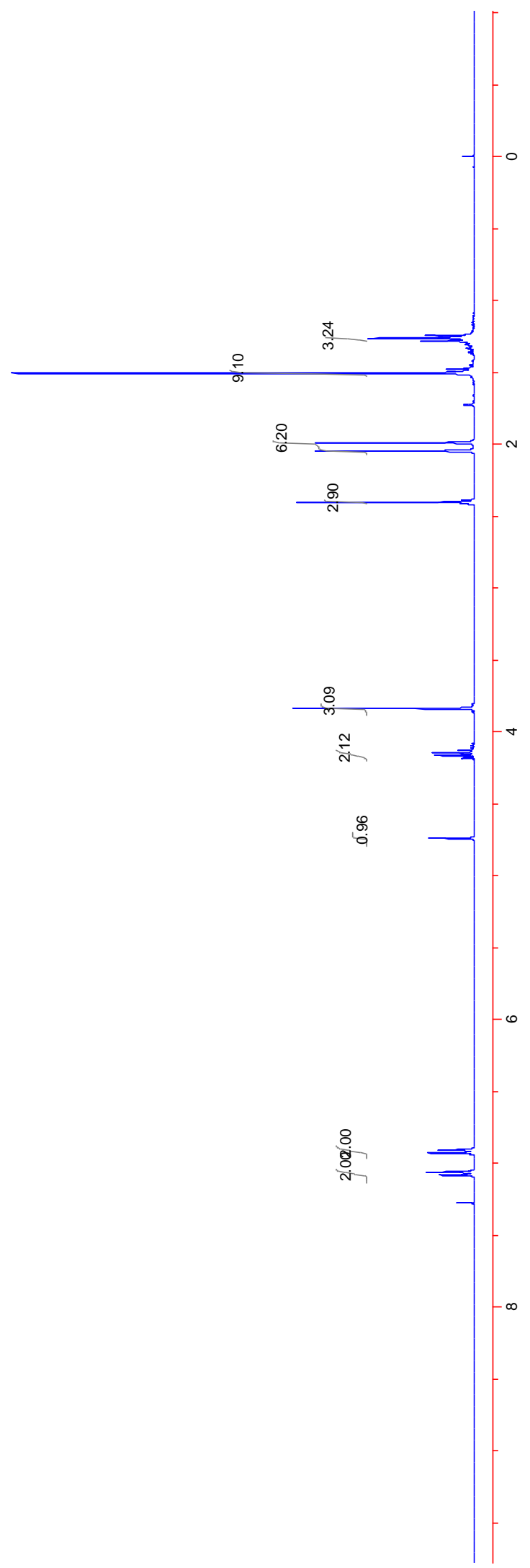

$8 \varepsilon 8^{\cdot} \varepsilon$

$62 l^{\circ} \mathrm{t}$

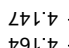

$\triangleright 9+\circ$

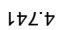

$895 \div-$

9LZ।

$\varepsilon 8 Z^{\prime}$

6Lt'เ

$809^{\circ}$.

1661

$6+02$

90t' 2

டเDZ

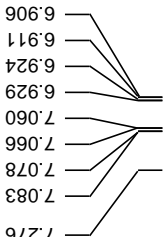

बัं 
${ }^{1}$ H NMR Spectra of diethyl 2-acetyl-3-( $p$-tolylamino)succinate 4

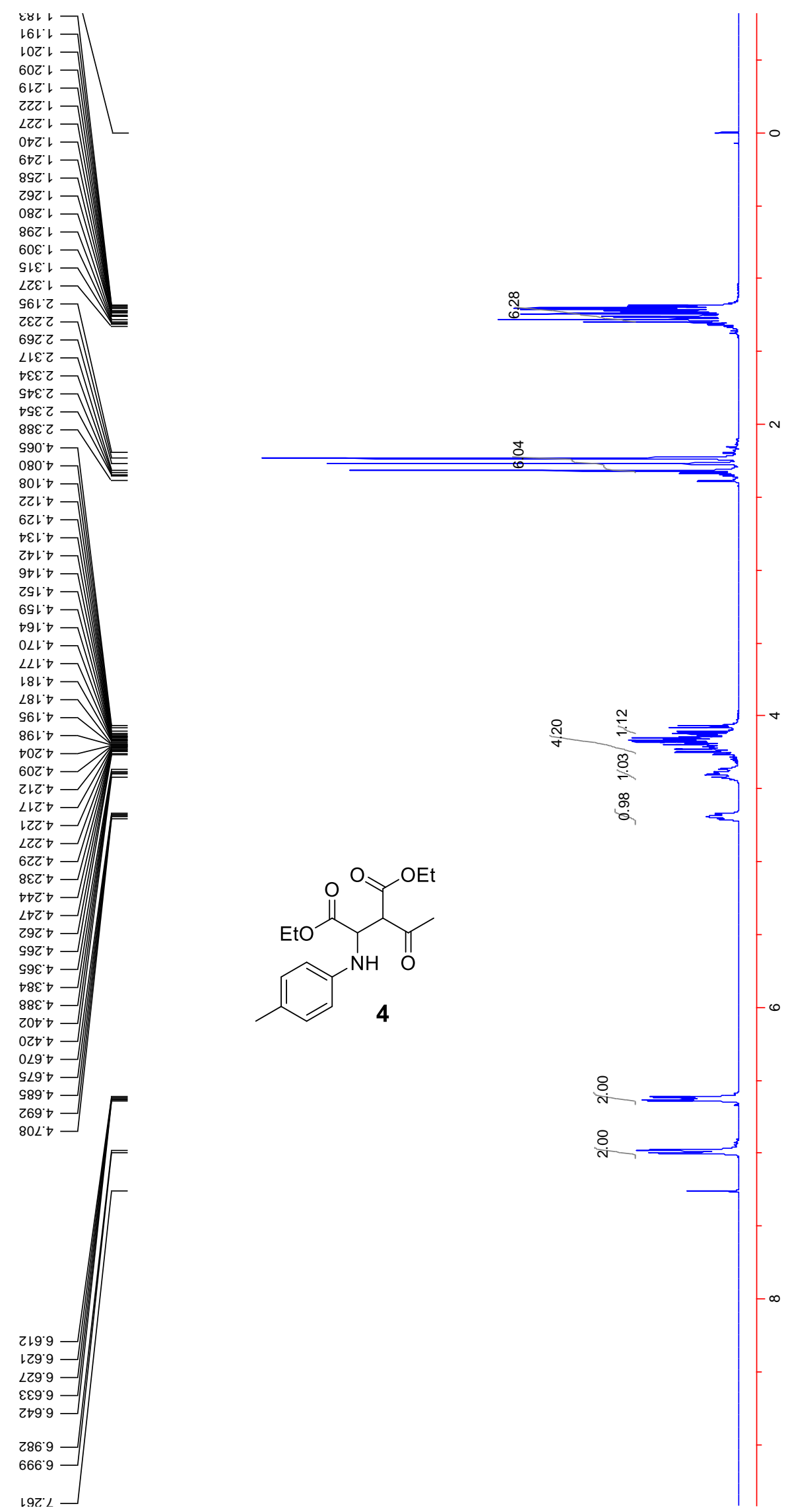


${ }^{13} \mathrm{C}$ NMR Spectra of diethyl 2-acetyl-3-(p-tolylamino)succinate 4
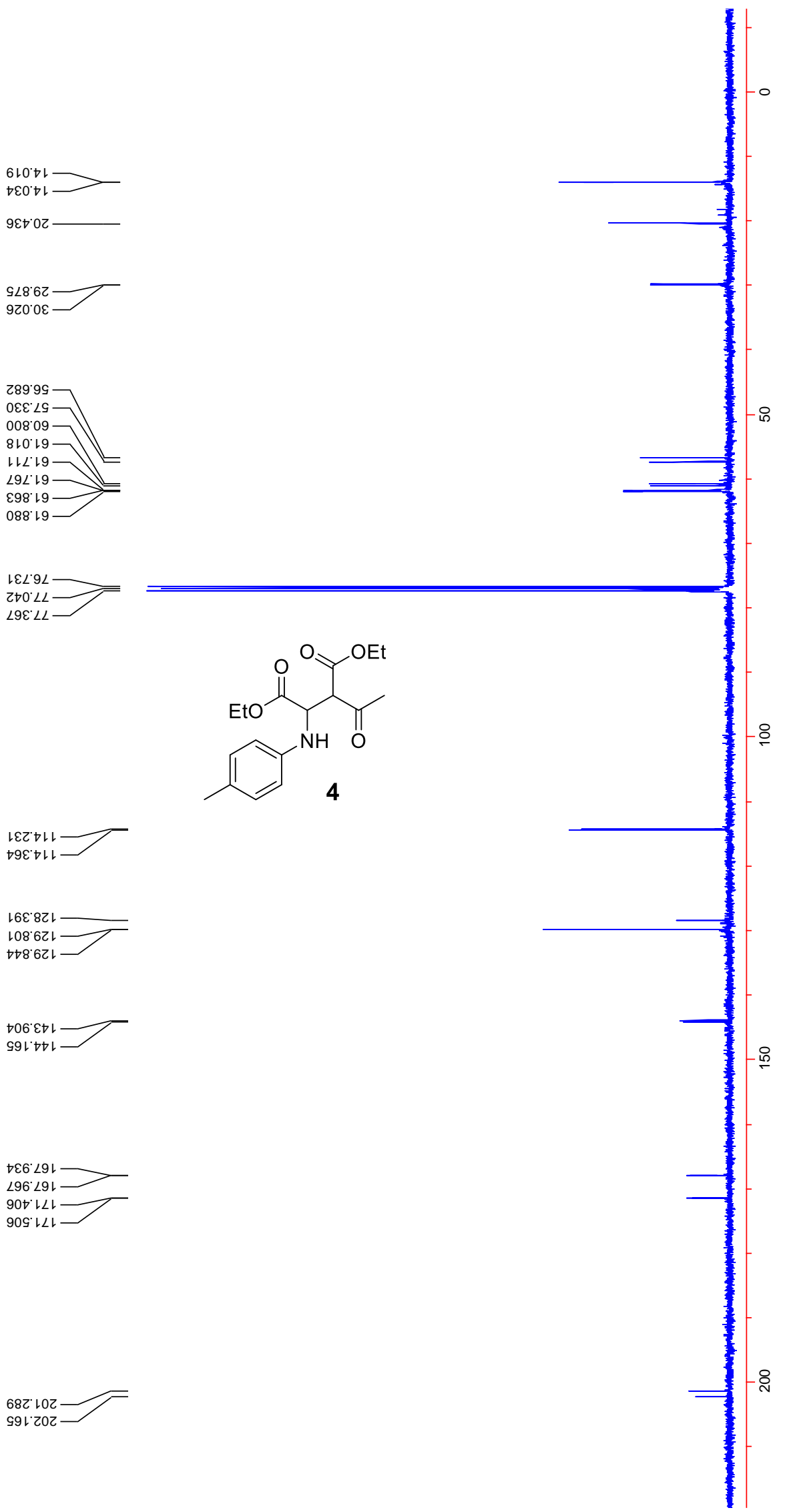

$2 \triangleright 0^{\circ} \angle L$

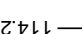

เย己๋เトレ

$16 \varepsilon \cdot 8 \mathrm{~L}$

108621

$\downarrow \nabla 8 \cdot 621$

†06. $\bullet$ เト

ง9เ・เト

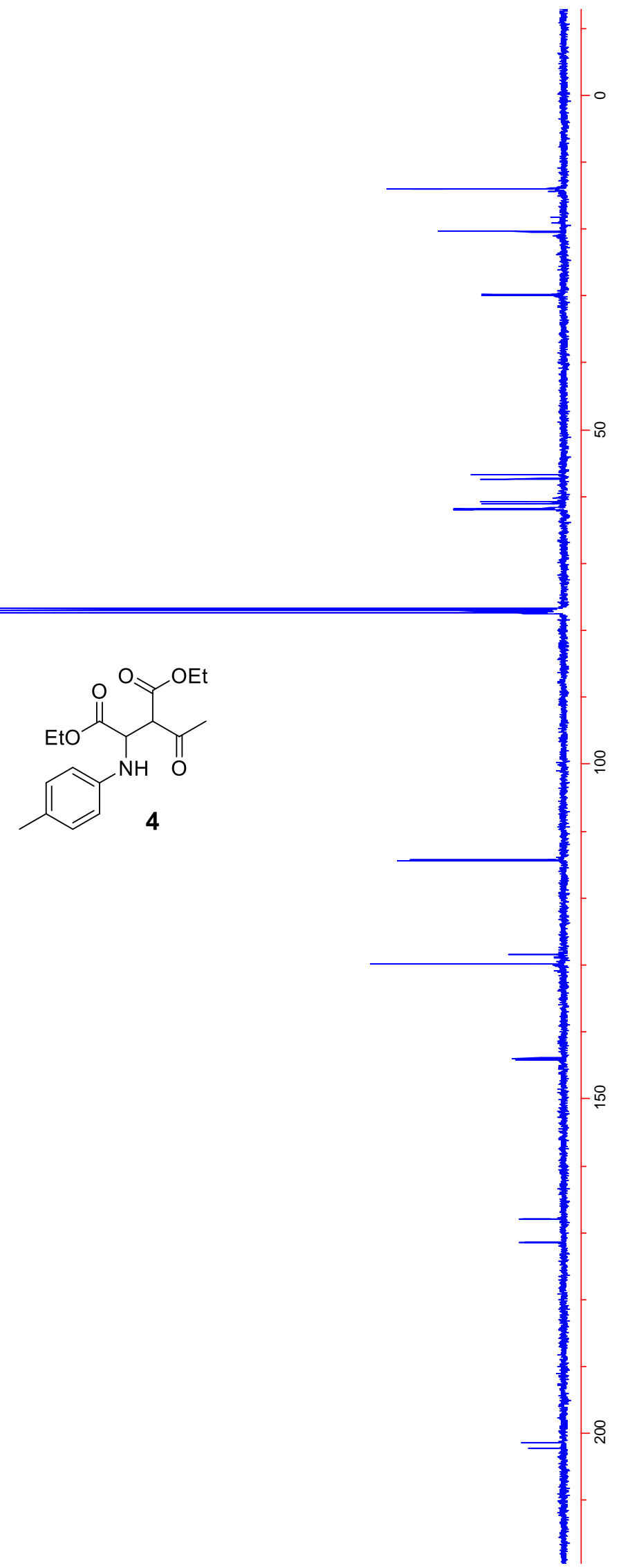

4

$\neg \varepsilon 6 \cdot \angle 91$

$\angle 96 \angle 91 \longrightarrow$

90t

$909 \cdot 1<1$

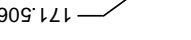

682 เ 02

S9. 202 
MS (EI) Spectra of diethyl 2-acetyl-3-(p-tolylamino)succinate 4

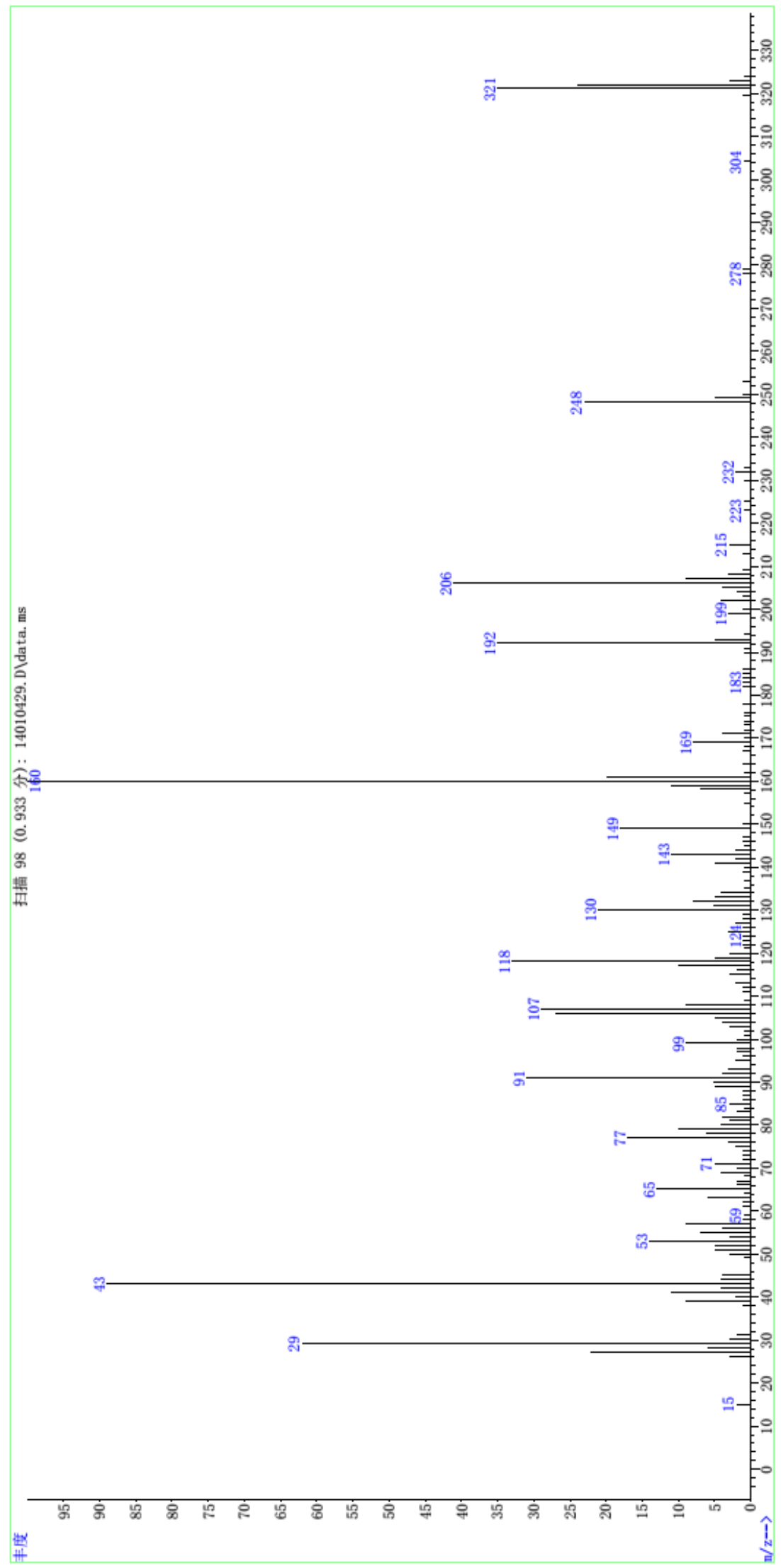

